\title{
On the geometric quantization of Jacobi manifolds
}

\author{
Manuel de Leóna) \\ Instituto de Matemáticas y Física Fundamental, \\ Consejo Superior de Investigaciones Científicas, Serrano 123, 28006 Madrid, Spain \\ Juan C. Marrero ${ }^{\mathrm{b})}$ and Edith Padrón ${ }^{\mathrm{c}}$ \\ Departamento de Matemática Fundamental, Facultad de Matemáticas, \\ Universidad de la Laguna, La Laguna, Tenerife, Canary Islands, Spain
}

(Received 23 June 1997; accepted for publication 7 July 1997)

The geometric quantization of Jacobi manifolds is discussed. A natural cohomology (termed Lichnerowicz-Jacobi) on a Jacobi manifold is introduced, and using it the existence of prequantization bundles is characterized. To do this, a notion of contravariant derivatives is used, in such a way that the procedure developed by Vaisman for Poisson manifolds is naturally extended. A notion of polarization is discussed and the quantization problem is studied. The existence of prequantization representations is also considered. (C) 1997 American Institute of Physics.

[S0022-2488(97)01212-7]

\section{INTRODUCTION}

The quantization of a classical system comes back to Dirac, and consists of associating with each classical observable $f$ on the phase space a Hermitian operator on some Hilbert space, in such a way that the Poisson bracket of two observables is associated (up to some constants) with the commutator of the operators. The geometric quantization theory is just a geometrization of this procedure. It was developed by Kostant ${ }^{1}$ and Souriau ${ }^{2}$ for symplectic manifolds, and it was later extended to Poisson manifolds by Vaisman. ${ }^{3}$ In an algebraic context, the geometric quantization of Poisson manifolds appears as a particular case of the quantization of Poisson algebras developed by Huebschmann. 4

The geometric theory proceeds in two steps. First, one associates to a symplectic manifold $M$ a prequantization bundle, that is, a complex line bundle $K$ over $M$. Next, one needs to restrict the space of observables, and one represents it irreducibly on a suitable Hilbert space which is constructed from $K$. The condition for the existence of a prequantization bundle is that the cohomology class of the symplectic form be integral. Indeed, the prequantization formula states that there exists a covariant derivative $\boldsymbol{\nabla}$ on the prequantization bundle such that the curvature of $\boldsymbol{\nabla}$ is (modulo some constants) the symplectic form. We refer to Refs. 5,6,7 as standard references, and to Ref. 8 for a recent survey.

For Poisson manifolds, Vaisman discovered that a more convenient geometric framework was to consider contravariant derivatives instead of covariant as in the case of symplectic forms. The reason is very clear, Poisson structures are defined by contravariant two-vectors instead of twoforms. So, Vaisman ${ }^{3}$ characterized the existence of prequantization bundles of a Poisson manifold $M$ by means of a natural cohomology of multivectors defined by Lichnerowicz, ${ }^{9}$ and termed Lichnerowicz-Poisson cohomology of $M$. This cohomology can be obtained as the cohomology of a subcomplex of the Chevalley-Eilenberg complex associated to the Lie algebra of functions $C^{\infty}(M, \mathbb{R})$.

The purpose of this paper is to extend these results to the context of Jacobi manifolds. As is well-known (see Refs. 10-12), Jacobi structures are the natural generalization of Poisson struc-

\footnotetext{
${ }^{a)}$ Electronic mail: mdeleon@pinar1.csic.es

${ }^{\mathrm{b})}$ Electronic mail: jcmarrer@ull.es

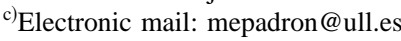


tures and in particular of symplectic, cosymplectic and Lie-Poisson structures. However, very interesting manifolds like contact and locally conformal symplectic (l.c.s.) manifolds are also Jacobi and they are not Poisson. A Jacobi bracket $\{$,$\} on a manifold M$ is in fact the most general local bilinear operator on the space of real functions $C^{\infty}(M, \mathrm{R})$ which is skew-symmetric and satisfies Jacobi's identity. Alternatively, a Jacobi structure on a manifold $M$ can be defined by a pair $(\Lambda, E)$, where $\Lambda$ is a two-vector and $E$ is a vector field on $M$ such that $[\Lambda, \Lambda]=2 E \wedge \Lambda$ and $[E, \Lambda]=0$. The link between both approaches is the formula $\{f, g\}=\Lambda(d f, d g)+f E(g)$ $-g E(f)$, for two functions $f$ and $g$ on $M$. In this paper we discuss first the prequantization problem in terms of an adequate cohomology (the Lichnerowicz-Jacobi cohomology), and later the quantization problem. It should be noticed that the problem of quantizing Jacobi manifolds has physical interest since the relation between them and BV-algebras (see Refs. 13-16).

The paper is organized as follows. In Sec. II we discuss some generalities on Jacobi manifolds. The main examples are given, and the characteristic foliation $\mathscr{F}$ is defined. Let us recall that if $(M, \Lambda, E)$ is a Jacobi manifold with Jacobi bracket $\{$,$\} then for every point x \in M, \mathscr{F}_{x}$ is generated for all the Hamiltonian vector fields evaluated at $x$. Since the Hamiltonian vector field associated with the function $f$ is $X_{f}=\#(d f)+f E$, where $\#: \Omega^{1}(M) \rightarrow \mathfrak{X}(M)$ is the $C^{\infty}(M, \mathrm{R})$-linear mapping induced by $\Lambda$, we get $\mathscr{F}_{x}=\#_{x}\left(T_{x}^{*} M\right)+\left\langle E_{x}\right\rangle$. We have that the even dimensional leaves of $\mathscr{F}$ are 1.c.s. manifolds, and the odd dimensional ones are contact manifolds, both with the induced Jacobi structure (see Ref. 10). We recall in this section the relationship between regular Jacobi manifolds and quantizable Poisson manifolds given in Ref. 17. The Lie algebroid structure on the jet bundle $J^{1}(M, \mathbb{R}) \cong T^{*} M \times \mathbb{R}$ of a Jacobi manifold $M$ introduced in Ref. 18 is also considered.

In a Jacobi manifold $(M, \Lambda, E)$ with Jacobi bracket $\{$,$\} , there are two different representations$ of the algebra of functions $C^{\infty}(M, \mathbb{R})$ on the module $C^{\infty}(M, \mathbb{R})$. The first one is defined by the Jacobi bracket, say $(f, g) \mapsto\{f, g\}$ and it leads to the usual Chevalley-Eilenberg cohomology. This cohomology has been studied by Lichnerowicz. ${ }^{12}$ The second one is defined by means of the Hamiltonian vector fields, say $(f, g) \mapsto X_{f}(g)$. For a Poisson manifold, they coincide, but for an arbitrary Jacobi manifold the second representation yields the so-called $H$-Chevalley-Eilenberg cohomology ( $H$ for Hamiltonian), which is discussed in Sec. III. The corresponding subcomplex of 1-differentiable cochains provides the Lichnerowicz-Jacobi cohomology, which can be identified with the cohomology $H_{L J}^{*}(M)$ of the complex $\left(\mathscr{V}^{*}(M) \oplus \mathscr{V}^{*-1}(M), \sigma\right)$, where $\mathscr{V}^{*}(M)$ $=\oplus_{k} \mathscr{V}^{k}(M), \mathscr{V}^{k}(M)$ is the space of $k$-vectors on $M$ and

$$
\sigma(P, Q)=(-[\Lambda, P]+k E \wedge P+\Lambda \wedge Q,[\Lambda, Q]-(k-1) E \wedge Q+[E, P])
$$

for $(P, Q) \in \mathscr{V}^{k}(M) \oplus \mathscr{V}^{k-1}(M)$. The cohomology provided by the subcomplex of $\left(\mathscr{V}^{*}(M)\right.$ $\left.\oplus \mathscr{V}^{*-1}(M), \sigma\right)$ consisting of pairs $(P, 0)$ with $P$ invariant by $E$ was previously studied in Refs. 19-21. An alternative way to introduce the Lichnerowicz-Jacobi cohomology which will be useful to introduce the Jacobi-Chern class of a complex line bundle over a Jacobi manifold is the following. Using the Lie algebroid structure on $J^{1}(M, \mathrm{R})$ we can define a representation of the Lie algebra $\left(\Omega^{1}(M) \times C^{\infty}(M, \mathbb{R}),\{\},\right)$ on the $\left(\Omega^{1}(M) \times C^{\infty}(M, \mathbb{R})\right)$-module $C^{\infty}(M, \mathrm{R})$. The cohomology of the subcomplex consisting of the $C^{\infty}(M, \mathbb{R})$-linear cochains is isomorphic to the Lichnerowicz-Jacobi cohomology. The relationship with the de Rham cohomology is also studied and we obtain a linear homomorphism $\mathbb{\#}: H_{d R}^{*}(M) \rightarrow H_{L J}^{*}(M)$ given by $\widetilde{\#}([\alpha])=[(\#(\alpha)$, $\left.\left.-\#\left(i_{E} \alpha\right)\right)\right]$.

In Sec. IV we introduce the notion of Jacobi-Chern class of a complex line bundle $K$ over a Jacobi manifold $(M, \Lambda, E)$ (see also Ref. 22). To do this, we first extend the concept of contravariant derivative given by Vaisman for Poisson manifolds to Jacobi manifolds. A contravariant derivative $D$ is defined as a derivation of sections with respect to pairs $(\alpha, f)$ consisting of a 1 -form $\alpha$ and a function $f$. We also define the curvature $C_{D}$ of $D$. The curvature $C_{D}$ defines a 
cohomology class $j c(K, \Lambda, E) \in H_{L J}^{2}(M)$, which is called the real Jacobi-Chern class of $K$. The Jacobi-Chern class is related with the usual real Chern class $c(K, \mathbb{R})$ of $K$ by the formula $\widetilde{\#}(c(K, \mathbb{R}))=j c(K, \Lambda, E)$.

Section $\mathrm{V}$ is devoted to a discussion on the prequantization of Jacobi manifolds. We say that a Jacobi manifold $(M, \Lambda, E)$ is quantizable if there exists a complex line bundle $\pi: K \rightarrow M$ over $M$ such that

$$
\{\widehat{f, g}\}=\hat{f} \circ \hat{g}-\hat{g} \circ \hat{f}, \quad f, g \in C^{\infty}(M, \mathbb{R})
$$

with $\hat{f} \in \operatorname{End}_{\mathrm{c}}(\Gamma(K))$ defined by $s \in \Gamma(K) \mapsto \hat{f}(s)=D_{j^{1} f} s+2 \pi i f s=D_{(d f, f)} s+2 \pi i f s$. Here, $\Gamma(K)$ is the space of cross sections of $\pi: K \rightarrow M$ and $D$ is a contravariant derivative on $K$. The main result of this section is a necessary and sufficient condition for a Jacobi manifold to be quantizable:

Theorem V.2: Let $(M, \Lambda, E)$ be a Jacobi manifold. Then, $M$ is quantizable if and only if there exist a vector field $A$, a real differentiable function $f$ and a closed 2-form $\Omega$ which represents an integral cohomology class of $M$ such that

(i) $\quad \widetilde{\#}(\Omega)=\sigma(A, f)$.

(ii) If $x$ is a point of $M$ and $E_{x}=0$ then $f(x)=1$.

(iii) If $x$ is a point of $M$ and $\omega$ is a l-form at $x$ such that $E_{x} \neq 0$ and $\#_{x}(\omega)=E_{x}$ then $f(x)$ $=\omega\left(A_{x}\right)+1$.

For a Poisson manifold we recover the result obtained by Vaisman. ${ }^{3}$ In particular, if $M$ is a symplectic manifold with symplectic 2 -form $\Phi$, we deduce that $M$ is quantizable is and only if $\Phi$ represents an integral cohomology class of $M$ (see Refs. 1-3). These examples and many others are discussed in Sec. VI. We study the case of Jacobi manifolds with 1.c.s. characteristic foliation and conclude that a 1.c.s. manifold is quantizable if and only if it is a quantizable symplectic manifold. For Jacobi manifolds with contact characteristic foliation, we deduce that they are always quantizable, so, in particular, every contact manifold is. The particular case of a regular Jacobi manifold and some interesting examples of Jacobi manifolds with mixed leaves (with nonpure characteristic foliation in our terminology) are also discussed.

The space of sections $\Gamma(K)$ of the quantum bundle $K$ of a quantizable Jacobi manifold $(M, \Lambda, E)$ is usually too big for quantization purposes, so that we have to introduce a polarization in order to reduce it. In Sec. VII we define a polarization of $M$ as a subspace $\mathscr{P}$ of $\Omega^{1}(M) \otimes \mathrm{C}$ such that $\mathscr{P} \times\{0\}$ is a subalgebra of $\left(\left(\Omega^{1}(M) \times C^{\infty}(M, \mathbb{R})\right) \otimes \mathrm{C},\{\},\right)$ and $\Lambda(\alpha, \beta)=0$, for $\alpha, \beta \in \mathscr{P}$. Here, the bracket $\{$,$\} is the natural extension of the bracket on \Omega^{1}(M) \times C^{\infty}(M, \mathbb{R})$. The subalgebra of the straightforwardly quantizable observables is then $P(\mathscr{P})=\left\{f \in C^{\infty}(M, \mathbb{R}) /\{(d f, f),(\alpha, 0)\} \in \mathscr{P}\right.$ $\times\{0\}$, for all $\alpha \in \mathscr{P}\}$. If we choose a Hermitian metric $h$ on $K$ and a quantization Hermitian contravariant derivative $D$, we can extend $D$ to the space of sections $\Gamma(K \otimes \mathscr{D})$ of $K \otimes \mathscr{D}$, where $\mathscr{D}$ is the complex line bundle of complex half-densities of $M$. If $M$ is compact, and we denote $\mathscr{H}_{0}=\left\{v \in \Gamma(K \otimes \mathscr{D}) /\right.$ for every $\left.\alpha \in \mathscr{P}, D_{(\alpha, 0)} v=0\right\}$, then $\mathscr{H}_{0}$ can be made a pre-Hilbert space with the scalar product

$$
\left\langle s_{1} \otimes \varrho_{1}, s_{2} \otimes \varrho_{2}\right\rangle=\int_{M} h\left(s_{1}, s_{2}\right) \varrho_{1} \bar{\varrho}_{2},
$$

where the bar denotes complex conjugation. Moreover, if $\hat{f}: \Gamma(K \otimes \mathscr{D}) \rightarrow \Gamma(K \otimes \mathscr{D})$ is the operator given by $\hat{f}(s \otimes \varrho)=D_{(d f, f)}(s \otimes \varrho)+2 \pi i f(s \otimes \varrho)$ then $\{\widehat{f, g}\}(s \otimes \varrho)=(\hat{f} \circ \hat{g}-\hat{g} \circ \hat{f})(s \otimes \varrho)$ and if, in addition, $f \in P(\mathscr{P})$ then $\hat{f}\left(\mathscr{H}_{0}\right) \subseteq \mathscr{H}_{0}$ and the operator $i \hat{f}: \mathscr{H}_{0} \rightarrow \mathscr{H}_{0}$ is Hermitian. The noncompact case is also considered. The obtained results in both cases extend those by Vaisman for 
Poisson manifolds. ${ }^{3}$ We end the section by discussing several examples of contact manifolds and Jacobi manifolds with nonpure characteristic foliation. We omit the case of symplectic manifolds since there exists a large literature on the subject.

In Sec. VIII we discuss the existence of (1-differentiable) prequantization representations for complex line bundles on Jacobi manifolds in terms of contravariant derivatives and of the real Jacobi-Chern class. The existence of these representations has been studied by Vaisman ${ }^{3}$ but using covariant derivatives. Our results extend the previous ones obtained by Urwin ${ }^{23}$ for symplectic manifolds and by Vaisman for Poisson manifolds. ${ }^{3}$

Finally, it should be noticed that an alternative approach to the quantization of Poisson manifolds was developed by Karasev ${ }^{24}$ and Weinstein ${ }^{25}$ by using the notion of symplectic groupoid (see also Refs. 26,27). The extension of the theory for Jacobi manifolds is a matter of obvious interest. Some work has been done by Dazord ${ }^{28}$ by introducing the notion of contact groupoid.

\section{JACOBI AND POISSON MANIFOLDS}

All the manifolds considered in this paper are assumed to be connected.

\section{A. Local Lie algebras and Jacobi manifolds}

A Jacobi structure on a $m$-dimensional manifold $M$ is a pair $(\Lambda, E)$, where $\Lambda$ is a 2-vector and $E$ a vector field on $M$ satisfying the following properties:

$$
[\Lambda, \Lambda]=2 E \wedge \Lambda, \quad \mathscr{L}_{E} \Lambda=[E, \Lambda]=0 .
$$

Here [,] denotes the Schouten-Nijenhuis bracket ${ }^{27,29}$ and $\mathscr{B}$ is the Lie derivative operator. The manifold $M$ endowed with a Jacobi structure is called a Jacobi manifold. A bracket of functions (the Jacobi bracket) is defined by

$$
\{f, g\}=\Lambda(d f, d g)+f E(g)-g E(f), \text { for all } f, g \in C^{\infty}(M, \mathbb{R}) .
$$

The Jacobi bracket $\{$,$\} is skew-symmetric, satisfies the Jacobi identity and$

$$
\text { support }\{f, g\} \subseteq(\text { support } f) \cap(\text { support } g) \text {. }
$$

Thus the space $C^{\infty}(M, \mathbb{R})$ of $C^{\infty}$ real-valued functions on $M$ endowed with the Jacobi bracket is $a$ local Lie algebra in the sense of Kirillov (see Ref. 30). Conversely, a structure of local Lie algebra on $C^{\infty}(M, \mathbb{R})$ defines a Jacobi structure on $M$ (see Refs. 11,30). If the vector field $E$ identically vanishes then $\{$,$\} is a derivation in each argument and, therefore, \{$,$\} defines a Poisson bracket on$ $M$. In this case, (1) reduces to $[\Lambda, \Lambda]=0$ and $(M, \Lambda)$ is a Poisson manifold. Jacobi and Poisson manifolds were introduced by Lichnerowicz ${ }^{9,12}$ (see also Refs. 29,31, and 27).

\section{B. Examples of Jacobi manifolds}

Examples of Poisson structures are symplectic and Lie-Poisson structures (see Refs. 9 and 32). Other examples of Poisson manifolds are the cosymplectic manifolds.

A cosymplectic manifold (see Refs. 33,34,35 and 17) is a triple $(M, \Phi, \eta)$, where $M$ is an odd dimensional manifold, $\Phi$ is a closed 2-form and $\eta$ is a closed 1-form on $M$ such that $\eta \Lambda \Phi^{m}$ is a volume form, with $\operatorname{dim} M=2 m+1$. If $b: \mathfrak{X}(M) \rightarrow \Omega^{1}(M)$ is the isomorphism of $C^{\infty}(M, \mathbb{R})$-modules from the space of the vector fields $\mathfrak{X}(M)$ on $M$ onto the space of 1-forms $\Omega^{1}(M)$ defined by $b(X)=i_{x} \Phi+\left(i_{x} \eta\right) \eta$, then the vector field $\xi=b^{-1}(\eta)$ is called the Reeb vector field of $M$. The Reeb vector field $\xi$ is characterized by the relations $i_{\xi} \Phi=0$ and $i_{\xi} \eta=1$. In particular, $\mathscr{L}_{\xi} \Phi=0$ and $\mathscr{L}_{\xi} \eta=0$. A 2 -vector $\Lambda$ on $M$ is defined by

$$
\Lambda(\alpha, \beta)=\Phi\left(b^{-1}(\alpha), b^{-1}(\beta)\right)=\Phi\left(b^{-1}(\alpha-\alpha(\xi) \eta), b^{-1}(\beta-\beta(\xi) \eta)\right),
$$


for all $\alpha, \beta \in \Omega^{1}(M)$. Thus $(M, \Lambda)$ becomes a Poisson manifold. Moreover, $\mathscr{L}_{\xi} \Lambda=0$. In canonical coordinates $\left\{q^{1}, \ldots, q^{m}, p_{i}, \ldots, p_{m}, z\right\}$, we have ${ }^{33,35}$

$$
\Phi=\sum_{i=1}^{m} d q^{i} \wedge d p_{i}, \quad \eta=d z, \quad \xi=\frac{\partial}{\partial z}, \quad \Lambda=\sum_{i=1}^{m} \frac{\partial}{\partial q^{i}} \wedge \frac{\partial}{\partial p_{i}} .
$$

Other interesting examples of Jacobi manifolds, which are not Poisson manifolds, are the contact manifolds and the locally conformal symplectic manifolds which we will describe below. Let $M$ be a $(2 m+1)$-dimensional manifold and $\theta$ a 1-form on $M$. We said that $\theta$ is a contact 1-form if $\theta \wedge(d \theta)^{m} \neq 0$ at every point. In such a case $(M, \theta)$ is termed a contact manifold (see, for example, Refs. 31,33 , and 34). A contact manifold $(M, \theta)$ is a Jacobi manifold. In fact, we define the 2-vector $\Lambda$ on $M$ by

$$
\Lambda(\alpha, \beta)=d \theta\left(b^{-1}(\alpha), b^{-1}(\beta)\right)
$$

for all $\alpha, \beta \in \Omega^{1}(M)$, where $b: \mathfrak{X}(M) \rightarrow \Omega^{1}(M)$ is the isomorphism of $C^{\infty}(M, \mathbb{R})$-modules given by $b(X)=i_{X} d \theta+\theta(X) \theta$. The vector field $E$ is just the Reeb vector field $\xi=b^{-1}(\theta)$ of $(M, \theta)$. It is characterized by the relations $i_{\xi} \theta=1$ and $i_{\xi} d \theta=0$. Moreover, around every point of $M$ there exist canonical coordinates $\left(t, q^{1}, \ldots, q^{m}, p_{1}, \ldots, p_{m}\right)$ such that (see Refs. 12, 33, and 34)

$$
\theta=d t-\sum_{i} p_{i} d q^{i}, \quad \Lambda=\sum_{i}\left(\frac{\partial}{\partial q^{i}}+p_{i} \frac{\partial}{\partial l}\right) \wedge \frac{\partial}{\partial p_{i}}, \quad E=\frac{\partial}{\partial t}
$$

An almost symplectic manifold is a pair $(M, \Phi)$, where $M$ is an even dimensional manifold and $\Phi$ is a nondegenerate 2 -form on $M$. An almost symplectic manifold is said to be locally conformal symplectic (l.c.s.) if for each point $x \in M$ there is an open neighborhood $U$ such that $d\left(e^{-\sigma} \Phi\right)=0$, for some function $\sigma: U \rightarrow \mathrm{R}$ (see, for example, Refs. 11, 17, and 36). So, $\left(U, e^{-\sigma} \Phi\right)$ is a symplectic manifold. If $U=M$ then $M$ is said to be a globally conformal symplectic (g.c.s.) manifold. An almost symplectic manifold $(M, \Phi)$ is 1.(g.)c.s. if and only if there exists a closed (exact) 1-form $\omega$ such that $d \Phi=\omega \wedge \Phi$. The 1-form $\omega$ is called the Lee 1-form of $M$. It is obvious that the 1.c.s. manifolds with Lee 1 -form identically zero are just the symplectic manifolds.

In a similar way that for contact manifolds, we define a 2-vector $\Lambda$ and a vector field $E$ on $M$ which are given by

$$
\Lambda(\alpha, \beta)=\Phi\left(b^{-1}(\alpha), b^{-1}(\beta)\right), \quad E=b^{-1}(\omega),
$$

for all $\alpha, \beta \in \Omega^{1}(M)$, where $b: \mathfrak{X}(M) \rightarrow \Omega^{1}(M)$ is the isomorphism of $C^{\infty}(M, \mathbb{R})$-modules defined by $b(X)=i_{x} \Phi$. Then $(M, \Lambda, E)$ is a Jacobi manifold. Notice that

$$
\omega(E)=0, \quad \mathscr{L}_{E} \omega=0, \quad \mathscr{L}_{E} \Phi=0 .
$$

Using the classical theorem of Darboux, around every point of $M$ there exist canonical coordinates $\left(q^{1}, \ldots, q^{m}, p_{1}, \ldots, p_{m}\right)$ and a local differentiable function $\sigma$ such that

$$
\begin{gathered}
\Phi=e^{\sigma} \sum_{i} d q^{i} \wedge d p_{i}, \quad \omega=d \sigma=\sum_{i}\left(\frac{\partial \sigma}{\partial q^{i}} d q^{i}+\frac{\partial \sigma}{\partial p_{i}} d p_{i}\right), \\
\Lambda=e^{-\sigma} \sum_{i}\left(\frac{\partial}{\partial q^{i}} \wedge \frac{\partial}{\partial p_{i}}\right), \quad E=e^{-\sigma} \sum_{i}\left(\frac{\partial \sigma}{\partial p_{i}} \frac{\partial}{\partial q^{i}}-\frac{\partial \sigma}{\partial q^{i}} \frac{\partial}{\partial p_{i}}\right) .
\end{gathered}
$$


Finally, a very simple but interesting Jacobi structure is that provided by a vector field $E$ on a manifold $M$. That is, the Jacobi structure is given by $(\Lambda=0, E)$. It should be remarked that this structure is closely related with Virasoro algebras (see Ref. 37).

\section{The characteristic foliation of a Jacobi manifold}

Let $(M, \Lambda, E)$ be a Jacobi manifold. Define a mapping \#: $\Omega^{1}(M) \rightarrow \mathfrak{X}(M)$ by

$$
(\#(\alpha))(\beta)=\Lambda(\alpha, \beta),
$$

for $\alpha, \beta \in \Omega^{1}(M)$. This mapping can be extended to a mapping, which we also denote by \#, from the space of $k$-forms $\Omega^{k}(M)$ on $M$ onto the space of $k$-vectors $\mathscr{T}^{k}(M)$ by putting

$$
\#(f)=f, \quad \#(\alpha)\left(\alpha_{1}, \ldots, \alpha_{k}\right)=(-1)^{k} \alpha\left(\#\left(\alpha_{1}\right), \ldots, \#\left(\alpha_{k}\right)\right),
$$

for all $f \in C^{\infty}(M, \mathbb{R}), \alpha \in \Omega^{k}(M)$ and $\alpha_{1}, \ldots, \alpha_{k} \in \Omega^{1}(M)$.

In the particular case of a cosymplectic manifold $M$ with Reeb vector field $\xi$, we have that $\#(\alpha)=-b^{-1}(\alpha)+\alpha(\xi) \xi$ for $\alpha \in \Omega^{1}(M)$. For a contact manifold with Reeb vector field $\xi$, we also deduce that $\#(\alpha)=-b^{-1}(\alpha)+\alpha(\xi) \xi$. For a 1.c.s. manifold, we obtain that $\#=-b^{-1}$.

If $f$ is a $C^{\infty}$ real-valued function on a Jacobi manifold $M$, the vector field $X_{f}$ defined by

$$
X_{f}=\#(d f)+f E
$$

is called the Hamiltonian vector field associated with $f$. It should be noticed that the Hamiltonian vector field associated with the constant function 1 is just $E$. A direct computation proves that (see Refs. 12 and 38)

$$
\left[X_{f}, X_{g}\right]=X_{\{f, g\}} .
$$

Now, for every $x \in M$, we consider the subspace $\mathscr{F}_{x}$ of $T_{x} M$ generated by all the Hamiltonian vector fields evaluated at the point $x$. In other words, $\mathscr{F}_{x}=\#_{x}\left(T_{x}^{*} M\right)+\left\langle E_{x}\right\rangle$. Since $\mathscr{F}$ is involutive, one easily follows that $\mathscr{F}$ defines a generalized foliation, which is called the characteristic foliation in Ref. 10. Moreover, the Jacobi structure of $M$ induces a Jacobi structure on each leaf. In fact, if $L$ is the leaf over a point $x$ of $M$ and $E_{x} \notin \operatorname{Im} \#_{x}$ then $L$ is a contact manifold with the induced Jacobi structure. If $E_{x} \in \operatorname{Im}_{x}, L$ is a 1.c.s. manifold (for a more detailed study of the characteristic foliation of a Jacobi manifold we refer to Ref. 10). If $M$ is a Poisson manifold then, from (4) and (6), we deduce that the characteristic foliation of $M$ is just the canonical symplectic foliation of $M$ (see Refs. 9 and 32).

\section{Regular Jacobi manifolds and quantizable Poisson manifolds}

A Jacobi manifold $(M, \Lambda, E)$ is said to be regular if the vector field $E$ is complete, $E \neq 0$ at every point and the 1-dimensional foliation defined by $E$ is regular in the sense of Palais. ${ }^{39}$ In such a case, the space of leaves $\bar{M}=M / E$ has a structure of differentiable manifold and the canonical projection $\pi: M \rightarrow \bar{M}$ is a fibration (surjective submersion). Moreover, we can define on $\bar{M}$ a 2-vector $\bar{\Lambda}$ by

$$
\bar{\Lambda}(\bar{\alpha}, \bar{\beta}) \circ \pi=A\left(\pi^{*} \bar{\alpha}, \pi^{*} \bar{\beta}\right), \quad \forall \bar{\alpha}, \bar{\beta} \in \Omega^{1}(\bar{M}) .
$$

Notice that, from (1), $\bar{\Lambda}$ is well-defined and $(\bar{M}, \bar{\Lambda})$ is a Poisson manifold (see Ref. 10). Next, we will relate the regular Jacobi manifolds with the quantizable Poisson manifolds (see Ref. 17).

We first recall the characterization of quantizable Poisson manifolds given by Vaisman. ${ }^{3} \mathrm{~A}$ Poisson manifold $(\bar{M}, \bar{\Lambda})$ is quantizable if and only if there exist a vector field $A$ and an integral closed 2-form $\bar{\Omega}$ on $\bar{M}$ such that 


$$
\bar{\Lambda}-\mathscr{L}_{\bar{A}} \bar{\Lambda}=\#(\bar{\Omega}),
$$

where \#: $\Omega^{2}(\bar{M}) \rightarrow \mathscr{V}^{2}(\bar{M})$ is the homomorphism given as in (4) and (5).

Now, suppose that $(\bar{M}, \bar{\Lambda})$ is a quantizable Poisson manifold and that $\bar{A}, \bar{\Omega}$ are a vector field and an integral closed 2-form on $\bar{M}$, respectively, satisfying (8). From the results of Ref. 40, there exists a principal circle bundle $\pi: M \rightarrow \bar{M}$ over $\bar{M}$ with connection form $\theta$ such that $\bar{\Omega}$ is the curvature for the connection $\theta$, that is, $\pi^{*} \bar{\Omega}=d \theta$.

In Ref. 17 we have proved that on $M$ there exists a Jacobi structure $(\Lambda, E)$ such that $(M, \Lambda, E)$ is a regular Jacobi manifold and the corresponding quotient Poisson manifold $M / E$ is just $(\bar{M}, \bar{\Lambda})$. Moreover,

$$
\theta(E)=1, \quad \mathscr{L}_{E} \theta=0 .
$$

In fact, $E$ is the infinitesimal generator of the action of $S^{1}$ on $M$ and $\Lambda$ is given by

$$
\Lambda=\bar{\Lambda}^{H}+E \wedge \bar{A}^{H},
$$

where $\bar{\Lambda}^{H}$ (respectively, $\bar{A}^{H}$ ) is the horizontal life to $M$ of $\bar{\Lambda}$ (respectively, $\bar{A}$ ) (see Remark II.1 below).

A converse of the above result is also proved. Namely, a compact regular Jacobi manifold $(M, \Lambda, E)$ endowed with a 1 -form $\theta$ satisfying (9) is the total space of a principal circle bundle over a quantizable Poisson manifold (see Ref. 17).

Remark II.1: Let $\pi: M \rightarrow \bar{M}$ be a principal circle bundle over a manifold $\bar{M}$ endowed with a connection form $\theta$. If $\bar{P}$ is a $k$-vector on $\bar{M}, k \geqslant 1$, we define the horizontal lift of $\bar{P}$ to $M$ as the $k$-vector $\bar{P}^{H}$ on $M$ characterized by the following conditions:

$$
\bar{P}^{H}\left(\pi^{*} \bar{\alpha}_{1}, \ldots, \pi^{*} \bar{\alpha}_{k}\right)=\bar{P}\left(\bar{\alpha}_{1}, \ldots, \bar{\alpha}_{k}\right) \circ \pi, \quad i_{\theta} \bar{P}^{H}=0,
$$

for all $\bar{\alpha}_{1}, \ldots, \bar{\alpha}_{k} \in \Omega^{1}(\bar{M})$.

\section{E. Lie algebroid of a Jacobi manifold}

A Lie algebroid structure on a differentiable vector bundle $\pi: K \rightarrow M$ is a pair that consists of a Lie algebra structure $\{$,$\} on the space \Gamma(K)$ of the global cross sections of $\pi: K \rightarrow M$ and a homomorphism of $C^{\infty}(M, \mathbb{R})$-modules $\varrho: \Gamma(K) \rightarrow \mathfrak{X}(M)$ such that

(i) $\quad \varrho:(\Gamma(K),\{\},) \rightarrow(\mathfrak{X}(M),[]$,$) is a Lie algebra homomorphism.$

(ii) For all $f \in C^{\infty}(M, \mathrm{R})$ and for all $s_{1}, s_{2} \in \Gamma(K)$ one has

$$
\left\{s_{1}, f s_{2}\right\}=f\left\{s_{1}, s_{2}\right\}+\left(\varrho\left(s_{1}\right)(f)\right) s_{2} .
$$

A triple $(K,\{\},, \varrho)$ is called a Lie algebroid over $M$ (see Refs. 27 and 41$)$.

Let $(M, \Lambda, E)$ be a Jacobi manifold. In Ref. 18, the authors obtain a Lie algebroid structure on the jet bundle $J^{1}(M, \mathbb{R})$ as follows. It is well-known that if $T^{*} M$ is the cotangent bundle of $M$, the space $J^{1}(M, \mathrm{R})$ can be identified with the product manifold $K=T^{*} M \times \mathrm{R}$ in such a sense that the space $\Gamma(K)$ of the global cross sections of the vector bundle $K=T^{*} M \times \mathrm{R} \rightarrow M$ can be identified with $\Omega^{1}(M) \times C^{\infty}(M, \mathrm{R})$. Now, we consider on $\Omega^{1}(M) \times C^{\infty}(M, \mathrm{R})$ the bracket $\{$,$\} given by (see$ Ref. 18)

$$
\begin{aligned}
\{(\alpha, f),(\beta, g)\}= & j^{1}\left(\mathscr{L}_{(\#(\alpha)+f E)} g-\mathscr{L}_{(\#(\beta)+g E)} f-\Lambda(\alpha, \beta)\right)+\left(\left(\mathscr{L}_{(\#(\alpha)+f E)}-i_{E} \alpha\right)(\beta-d g)\right. \\
& \left.-\left(\mathscr{L}_{(\#(\beta)+g E)}-i_{E} \beta\right)(\alpha-d f), 0\right)=\left(\mathscr{D}_{\#(\alpha)} \beta-\mathscr{L}_{\#(\beta)} \alpha-d(\Lambda(\alpha, \beta))+f \mathscr{D}_{E} \beta\right. \\
& \left.-g \mathscr{L}_{E} \alpha-i_{E}(\alpha \wedge \beta), \alpha(\#(\beta))+\#(\alpha)(g)-\#(\beta)(f)+f E(g)-g E(f)\right),
\end{aligned}
$$


where $j^{1}: C^{\infty}(M, \mathbb{R}) \rightarrow \Omega^{1}(M) \times C^{\infty}(M, \mathbb{R})$ is the prolongation mapping defined by

$$
j^{1}(f)=(d f, f) .
$$

We have (see Ref. 18)

Theorem II.2: Let $(M, \Lambda, E)$ be a Jacobi manifold and $\left\{\right.$, the bracket on $\Omega^{1}(M) \times C^{\infty}(M, \mathbb{R})$ defined by (12). Then, the triple $\left(T^{*} M \times \mathbb{R},\{\},,(\#, E)\right)$ is a Lie algebroid over $M$, where $(\#, E): \Omega^{1}(M) \times C^{\infty}(M, \mathbb{R}) \rightarrow \mathfrak{X}(M)$ is the homomorphism of $C^{\infty}(M, \mathbb{R})$-modules given by

$$
(\#, E)(\alpha, f)=\#(\alpha)+f E .
$$

Moreover, if we consider on $C^{\infty}(M, \mathbb{R})$ the Jacobi bracket then the prolongation mapping

$$
j^{1}: C^{\infty}(M, \mathbb{R}) \rightarrow \Omega^{1}(M) \times C^{\infty}(M, \mathbb{R}), \quad f \rightarrow j^{1} f=(d f, f)
$$

is a Lie algebra homomorphism.

Remark II.3: (i) If $\mathfrak{X}_{H}(M)$ is the Lie algebra of the Hamiltonian vector fields, it is clear that $(\#, E)\left(j^{1}\left(C^{\infty}(M, \mathrm{R})\right)\right)=\mathfrak{X}_{H}(M)$.

(ii) In the particular case when $M$ is a Poisson manifold we recover, by projection, the usual Lie algebroid structure on the vector bundle $\pi: T^{*} M \rightarrow M$ (see Refs. 29, 42, 43).

\section{H-CHEVALLEY-EILENBERG COHOMOLOGY OF A JACOBI MANIFOLD}

Let $(M, \Lambda, E)$ be a Jacobi manifold and $\{$,$\} the Jacobi bracket. We consider the cohomology of$ the Lie algebra $\left(C^{\infty}(M, \mathbb{R}),\{\},\right)$ relative to the representation defined by the Hamiltonian vector fields, that is, to the representation given by

$$
C^{\infty}(M, \mathbb{R}) \times C^{\infty}(M, \mathbb{R}) \rightarrow C^{\infty}(M, \mathbb{R}), \quad(f, g) \rightarrow X_{f}(g) .
$$

This cohomology is denoted by $H_{H C E}^{*}(M)$ and it is called the H-Chevalley-Eilenberg cohomology associated to $M$ (see Refs. 20,21). In fact, if $C_{H C E}^{k}(M)$ is the real vector space of the $k$-linear skew-symmetric mappings $c^{k}: C^{\infty}(M, \mathbb{R}) \times \cdots\left(k \cdots \times C^{\infty}(M, \mathbb{R}) \rightarrow C^{\infty}(M, \mathbb{R})\right.$ then

$$
H_{H C E}^{k}(M)=\frac{\operatorname{ker}\left\{\partial_{H}: C_{H C E}^{k}(M) \rightarrow C_{H C E}^{k+1}(M)\right\}}{\operatorname{Im}\left\{\partial_{H}: C_{H C E}^{k-1}(M) \rightarrow C_{H C E}^{k}(M)\right\}},
$$

where $\partial_{H}: C_{H C E}^{r}(M) \rightarrow C_{H C E}^{r+1}(M)$ is the linear differential operator defined by

$$
\begin{aligned}
\left(\partial_{H} c^{r}\right)\left(f_{0}, \ldots, f_{r}\right)= & \sum_{i=0}^{r}(-1)^{i} X_{f_{i}}\left(c^{r}\left(f_{0}, \ldots, \hat{f}_{i}, \ldots, f_{r}\right)\right) \\
& +\sum_{i<j}(-1)^{i+j} c^{r}\left(\left\{f_{i}, f_{j}\right\}, f_{0}, \ldots, \hat{f}_{i}, \ldots, \hat{f}_{j}, \ldots, f_{r}\right)
\end{aligned}
$$

for $c^{r} \in C_{H C E}^{r}(M)$ and $f_{0}, \ldots, f_{r} \in C^{\infty}(M, \mathbb{R})$.

Notice that for a Poisson manifold, $H_{H C E}^{*}(M)$ is the Chevalley-Eilenberg cohomology of the Lie algebra $\left(C^{\infty}(M, \mathbb{R}),\{\},\right)$ (see Ref. 9). However, for arbitrary Jacobi manifolds the ChevalleyEilenberg cohomology (which is defined with respect to the representation given by the Jacobi bracket $^{12}$ ) does not coincide in general with the $H$-Chevalley-Eilenberg cohomology defined above.

Now, we will study the cohomology of the subcomplex of the 1-differentiable cochains. A $k$-cochain $c^{k} \in C_{H C E}^{k}(M)$ is said to be 1-differentiable if it is defined by a linear differential operator of order 1 . If $\mathscr{V}^{r}(M)$ is the space of $r$-vectors on $M$ then we can identify the space 
$\mathscr{V}^{k}(M) \oplus \mathscr{V}^{k-1}(M)$ with the space of all 1-differentiable $k$-cochains $C_{H C E-1 \text { diff }}^{k}(M)$ as follows (see, for instance, Ref. 9): define $j_{k}: \mathscr{V}^{k}(M) \oplus \mathscr{V}^{k-1}(M) \rightarrow C_{H C E}^{k}(M)$ the monomorphism given by

$$
j_{k}(P, Q)\left(f_{1}, \ldots, f_{k}\right)=P\left(d f_{1}, \ldots, d f_{k}\right)+\sum_{q=1}^{k}(-1)^{q+1} f_{q} Q\left(d f_{1}, \ldots, \widehat{d f_{q}}, \ldots, d f_{k}\right) .
$$

Then, $j_{k}\left(\mathscr{V}^{k}(M) \oplus \mathscr{V}^{k-1}(M)\right)=C_{H C E 1-\text { diff }}^{k}(M)$ which implies that the spaces $\mathscr{T}^{k}(M)$ $\oplus \mathscr{V}^{k-1}(M)$ and $C_{H C E 1-\operatorname{diff}}^{k}(M)$ are isomorphic.

On the other hand, if $\widetilde{P} \in C_{H C E 1-\operatorname{diff}}^{k}(M)$ then $\partial_{H} \widetilde{P} \in C_{H C E 1-\operatorname{diff}}^{k+1}(M)$. Thus, we have the corresponding subcomplex $\left(C_{H C E 1-\text { diff }}^{*}(M), \partial_{H \mid C_{H C E 1-\text { diff }}^{*}(M)}\right)$ of the $H$-Chevalley-Eilenberg complex whose cohomology $H_{H C E 1-\operatorname{diff}}^{*}(M)$ will be called the 1-differentiable H-ChevalleyEilenberg cohomology of $M$. Moreover, using (15), (16) and the properties of the SchoutenNijenhuis bracket, we can prove that

$$
\partial_{H}\left(j_{k}(P, Q)\right)=j_{k+1}(\sigma(P, Q))
$$

where

$$
\sigma(P, Q)=(-[\Lambda, P]+k E \wedge P+\Lambda \wedge Q,[\Lambda, Q]-(k-1) E \wedge Q+[E, P]) .
$$

The last equation defines a mapping $\sigma: \mathscr{V}^{k}(M) \oplus \mathscr{V}^{k-1}(M) \rightarrow \mathscr{V}^{k+1}(M) \oplus \mathscr{V}^{k}(M)$ which is in fact a differential operator that verifies $\sigma^{2}=0$. Thus we have a complex $\left(\mathscr{V}^{*}(M) \oplus \mathscr{V}^{*-1}(M), \sigma\right)$ whose cohomology will be called the Lichnerowicz-Jacobi cohomology (LJ-cohomology) of $M$ and denoted by $H_{L J}^{*}(M)$. This cohomology is a generalization of the Lichnerowicz-Jacobi cohomology introduced in Refs. 19-21. In fact, the former one is the cohomology of the subcomplex of the pairs $(P, 0)$, where $P$ is invariant by $E$. For this reason, we retain the name.

Notice that the mappings $j_{k}: \mathscr{V}^{k}(M) \oplus \mathscr{V}^{k-1}(M) \rightarrow C_{H C E}^{k}(M)$ given by (16) induce an isomorphism between the complexes $\left(\mathscr{V}^{*}(M) \oplus \mathscr{V}^{*-1}(M), \sigma\right)$ and $\left(C_{H C E 1-\operatorname{diff}}^{*}(M)\right.$, $\left(\partial_{H}\right)_{H C E 1-\text { diff }}(M)$ and therefore the corresponding cohomologies are isomorphic.

Remark III. 1: If $\widetilde{\sigma}$ denotes the cohomology operator in the 1-differentiable ChevalleyEilenberg subcomplex then (see Ref. 12)

$$
\hat{\sigma}(P, Q)=(-[\Lambda, P]+(k-1) E \wedge P+\Lambda \wedge Q,[\Lambda, Q]-(k-2) E \wedge Q+[E, P]),
$$

for $(P, Q) \in \mathscr{V}^{k}(M) \oplus \mathscr{V}^{k-1}(M)$. Thus, from (18), we deduce that the 1-differentiable $H$-Chevalley-Eilenberg cohomology (that is, the LJ-chomology) does not coincide in general with the 1-differentiable Chevalley-Eilenberg cohomology.

Now, we define a natural structure of $\left(\Omega^{1}(M) \times C^{\infty}(M, \mathbb{R})\right)$-module on $C^{\infty}(M, \mathbb{R})$ putting

$$
(\alpha, f) g=(\#, E)(\alpha, f)(g),
$$

where $(\#, E): \Omega^{1}(M) \times C^{\infty}(M, \mathrm{R}) \rightarrow \mathfrak{X}(M)$ is the homomorphism given by (13). If $\{$,$\} is the$ bracket on $\Omega^{1}(M) \times C^{\infty}(M, R)$ defined by (12) then, since $(\#, E):\left(\Omega^{1}(M)\right.$ $\left.\times C^{\infty}(M, \mathbb{R}),\{\},\right) \rightarrow(\mathfrak{X}(M),[]$,$) is a Lie algebra homomorphism (see Theorem II.2), we have a$ representation of the Lie algebra $\left(\Omega^{1}(M) \times C^{\infty}(M, \mathbb{R}),\{\},\right)$ onto the module $C^{\infty}(M, \mathbb{R})$, and we can define the corresponding differential complex and the corresponding cohomology. In this complex the $k$-cochains are the R-linear skew-symmentric mappings $\hat{R}:\left(\Omega^{1}(M) \times C^{\infty}(M, \mathbb{R})\right)^{k}$ $\rightarrow C^{\infty}(M, \mathbb{R})$ and the cohomology operator $\widetilde{\partial}$ is given by 


$$
\begin{aligned}
(\widetilde{\partial R})\left(\left(\alpha_{0}, f_{0}\right), \ldots,\left(\alpha_{k}, f_{k}\right)\right)= & \sum_{i=0}^{k}(-1)^{i}(\#, E)\left(\alpha_{i}, f_{i}\right)\left(\widetilde{R}\left(\left(\alpha_{0}, f_{0}\right), \ldots,\left(\widehat{\alpha_{i}, f_{i}}\right), \ldots,\left(\alpha_{k}, f_{k}\right)\right)\right) \\
& +\sum_{i<j}(-1)^{i+j} \widetilde{R}\left(\left\{\left(\alpha_{i}, f_{i}\right),\left(\alpha_{j}, f_{j}\right)\right\},\right. \\
& \left.\left(\alpha_{0}, f_{0}\right), \ldots,\left(\widehat{\alpha_{i}, f_{i}}\right), \ldots,\left(\widehat{\alpha_{i}, f_{j}}\right), \ldots,\left(\alpha_{k}, f_{k}\right)\right) .
\end{aligned}
$$

We can consider the subcomplex of those cochains which are $C^{\infty}(M, \mathrm{R})$-linear. The cohomology of this subcomplex is also isomorphic to the $L J$-cohomology. An isomorphism is induced by the mapping $\quad(P, Q) \mapsto(\widetilde{P, Q})$, where $\quad(P, Q) \in \mathscr{T}^{k}(M) \oplus \mathscr{V}^{k-1}(M) \quad$ and $\quad(\widetilde{P, Q}):\left(\Omega^{1}(M)\right.$ $\left.\times C^{\infty}(M, \mathrm{R})\right)^{k} \rightarrow C^{\infty}(M, \mathrm{R})$ is the $C^{\infty}(M, \mathbb{R})$-linear mapping defined by

$$
(\widetilde{P, Q})\left(\left(\alpha_{t}, f_{1}\right), \ldots,\left(\alpha_{k}, f_{k}\right)\right)=P\left(\alpha_{1}, \ldots, \alpha_{k}\right)+\sum_{q=1}^{k}(-1)^{q+1} f_{q} Q\left(\alpha_{1}, \ldots \widehat{\alpha_{q}} \ldots, \alpha_{k}\right) .
$$

In fact, we can prove that

$$
\widetilde{\partial}(\widetilde{P, Q})=\sigma(\widetilde{P, Q}) .
$$

Finally, we will show the relation between the de Rham cohomology and the LJ-cohomology. Denote by \#: $\Omega^{k}(M) \rightarrow \mathscr{V}^{k}(M)$ the homomorphism of $C^{\infty}(M, \mathbb{R})$-modules defined in (4) and (5). We have (see Refs. 19-21):

$$
\mathscr{L}_{E}(\#(\alpha))=\#\left(\mathscr{L}_{E} \alpha\right), \quad-[\Lambda, \#(\alpha)]+k E \wedge \#(\alpha)=-\#(d \alpha)+\#\left(i_{E} \alpha\right) \wedge \Lambda,
$$

for all $\alpha \in \Omega^{k}(M)$. Using these results and (18), we deduce

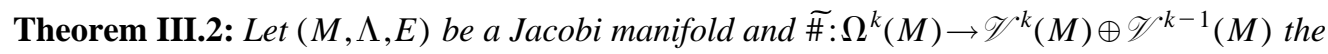
homomorphism of $C^{\infty}(M, \mathbb{R})$-modules given by

$$
\widetilde{\#}(\alpha)=\left(\#(\alpha),-\#\left(i_{E} \alpha\right)\right)
$$

for all $\alpha \in \Omega^{k}(M)$. Then $\widetilde{\#}$ induces a homomorphism of complexes $\widetilde{\#}:\left(\Omega^{*}(M), d\right) \rightarrow\left(\mathscr{V}^{*}(M)\right.$ $\left.\oplus \mathscr{V}^{*-1}(M),-\sigma\right)$. Thus if $H_{d R}^{*}(M)$ is the de Rham cohomology of $M$, we have the corresponding homomorphism in cohomology $\widetilde{\#}: H_{d R}^{*}(M) \rightarrow H_{L J}^{*}(M)$.

Remark III.3: If $(M, \Lambda)$ is a Poisson manifold we can define the linear differential operator $\widetilde{\sigma}: \mathscr{V}^{k}(M) \rightarrow \mathscr{V}^{k+1}(M)$ by $\bar{\sigma}(P)=-[\Lambda, P]$. Since $\bar{\sigma}^{2}=0, \widetilde{\sigma}$ defines a cohomology which is called the Lichnerowicz-Poisson cohomology (LP-cohomology) for the Poisson manifold $M$ (see Ref. 9). We will denote by $H_{L P}^{*}(M)$ the LP-cohomology. Using (22), we obtain that $\widetilde{\sigma}^{\circ} \#=-\#$ ${ }^{\circ} d$ and therefore we have an induced homomorphism in cohomology $\#: H_{d R}^{*}(M) \rightarrow H_{L P}^{*}(M)$. On the other hand, from (18), we deduce that the LP-cohomology of $M$ is isomorphic to the cohomology of the subcomplex of the LJ-complex consisting of the pairs $(P, 0)$. Consequently, using Theorem III.2 we obtain the following commutative diagram: 


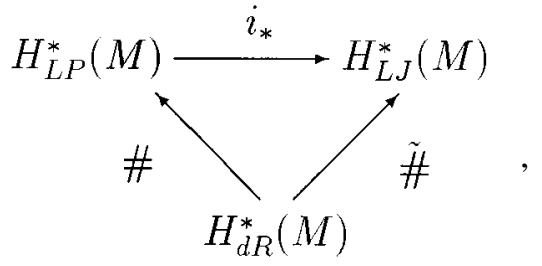

where $i_{*}$ is the induced homomorphism by the canonical inclusion $P \mapsto(P, 0)$.

\section{JACOBI-CHERN CLASS OF A COMPLEX LINE BUNDLE OVER A JACOBI MANIFOLD}

Let $(M, \Lambda, E)$ be a Jacobi manifold and $\pi: K \rightarrow M$ a complex line bundle over $M$. Denote by $\Gamma(K)$ the space of cross sections of $\pi: K \rightarrow M$ and, by $\operatorname{End}_{\mathrm{c}}(\Gamma(K))$ the space of the C-linear endomorphisms of $\Gamma(K)$.

Definition IV.1: A contravariant derivative $D$ on $\pi: K \rightarrow M$ is a mapping $D: \Omega^{1}(M)$ $\times C^{\infty}(M, \mathbb{R}) \rightarrow \operatorname{End}_{\mathrm{c}}(\Gamma(K))$ which satisfies the following conditions:

$$
\begin{gathered}
D_{(\alpha+\beta, f+g)}=D_{(\alpha, f)}+D_{(\beta, g)}, \quad D_{(g \alpha, g f)}=g D_{(\alpha, f)}, \\
D_{(\alpha, f)} g s=g D_{(\alpha, f)} s+(\#, E)(\alpha, f)(g) s,
\end{gathered}
$$

for all $\alpha \in \Omega^{1}(M), f, g \in C^{\infty}(M, \mathrm{R})$ and $s \in \Gamma(K)$.

This definition is a natural extension of the one given by Vaisman for Poisson manifolds (see Ref. 3, 27). In fact, if $(M, \Lambda)$ is a Poisson manifold and $D: \Omega^{1}(M) \times C^{\infty}(M, \mathrm{R}) \rightarrow \operatorname{End}_{\mathrm{c}}(\Gamma(K))$ is a contravariant derivative on a complex line bundle $\pi: K \rightarrow M$, then the mapping

$$
\widetilde{D}: \Omega^{1}(M) \rightarrow \operatorname{End}_{\mathrm{c}}(\Gamma(K)) \quad \widetilde{D}_{\alpha}=D_{(\alpha, 0)}
$$

defines a contravariant derivative in the sense of Vaisman. Conversely, if $\widetilde{D}: \Omega^{1}(M) \rightarrow E n d_{\mathrm{e}}(\Gamma(K))$ is a contravariant derivative in the sense of Vaisman then the mapping

$$
D: \Omega^{1}(M) \times C^{\infty}(M, \mathbb{R}) \rightarrow \operatorname{End}_{\mathrm{c}}(\Gamma(K)) \quad D_{(\alpha, f)}=\widetilde{D}_{\alpha}
$$

satisfies (24).

Let $(M, \Lambda, E)$ be a Jacobi manifold and $h$ a Hermitian metric on the complex line bundle $\pi: K \rightarrow M$. A contravariant derivative $D$ on $\pi: K \rightarrow M$ is said to be Hermitian (or compatible with $h)$ if

$$
(\#, E)(\alpha, f)\left(h\left(s_{1}, s_{2}\right)\right)=h\left(D_{(\alpha, f)} s_{1}, s_{2}\right)+h\left(s_{1}, D_{(\alpha, f)} s_{2}\right),
$$

for all $\alpha \in \Omega^{1}(M), f \in C^{\infty}(M, \mathbb{R})$ and $s_{1}, s_{2} \in \Gamma(K)$.

For instance, if $\boldsymbol{\nabla}$ is a usual (Hermitian) covariant derivative on $\pi: K \rightarrow M$ and we put $D_{(\alpha, f)}=\nabla_{(\#, E)(\alpha, f)}$, we obtain a (Hermitian) contravariant derivative. This remark shows that (Hermitian) contravariant derivatives always exist.

Next, we will introduce the definition of curvature of a contravariant derivative.

Definition IV.2: Let $\pi: K \rightarrow M$ be a complex line bundle over a Jacobi manifold $M$ and $D$ a contravariant derivative on $\pi: K \rightarrow M$. The curvature of $D$ is the mapping $C_{D}:\left(\Omega^{1}(M)\right.$ $\left.\times C^{\infty}(M, \mathrm{R})\right) \times\left(\Omega^{1}(M) \times C^{\infty}(M, \mathrm{R})\right) \times \Gamma(K) \rightarrow \Gamma(K)$ given by

$$
C_{D}((\alpha, f),(\beta, g))(s)=\left(D_{(\alpha, f)} \circ D_{(\beta, g)}-D_{(\beta, g)}^{\circ} D_{(\alpha, f)}-D_{\{(\alpha, f),(\beta, g)\}}\right) s
$$


for all $(\alpha, f),(\beta, g) \in \Omega^{1}(M) \times C^{\infty}(M, \mathbb{R})$ and $s \in \Gamma(K)$.

Using (24), (26) and Theorem II.2, we deduce that $C_{D}$ is trilinear over $C^{\infty}(M, \mathbb{R})$ and

$$
C_{D}((\alpha, f),(\beta, g))(s)=-C_{D}((\beta, g),(\alpha, f))(s) .
$$

Thus, from the results in Sec. III, we have that there exist a globally defined complex 2-vector $P_{C_{D}}=P_{1}+i P_{2}$ and a globally defined complex vector field $Y_{C_{D}}=Y_{1}+i Y_{2}$ such that

$$
C_{D}((\alpha, f),(\beta, g))(s)=\left(\left(\widetilde{P_{C_{D}}, Y_{C_{D}}}\right)((\alpha, f),(\beta, g))\right) s,
$$

where $\left(\widetilde{P_{C_{D}}, Y_{C_{D}}}\right)=\left(\widetilde{P_{1}, Y_{1}}\right)+i\left(\widetilde{P_{2}, Y_{2}}\right)$ and $\left(\widetilde{P_{i}, Y_{i}}\right)$ are defined by $(20)$.

Now, let $\sigma$ be the LJ-cohomology operator (see (18)). If $P=P_{1}+i P_{2}$ (respectively, $Q=Q_{1}$ $\left.+i Q_{2}\right)$ is a complex $k$-vector (respectively, $(k-1)$-vector) then we can define $\sigma(P, Q)$ by linearity as follows

$$
\sigma(P, Q)=\sigma\left(P_{1}, Q_{1}\right)+i \sigma\left(P_{2}, Q_{2}\right) .
$$

It is clear that $\sigma^{2}=0$ and, therefore, we obtain the corresponding cohomology which will be denoted by $H_{C L J}^{*}(M)$. Moreover, we prove the following.

Theorem IV.3: Let $\pi: K \rightarrow M$ be a complex line bundle over a Jacobi manifold M. Suppose that $D$ is a contravariant derivative on $\pi: K \rightarrow M$ with curvature $C_{D}$. Then:

(i) The pair $\left(P_{C_{D}}, Y_{C_{D}}\right)$ defines a cohomology class in $H_{C L J}^{2}(M)$.

(ii) The cohomology class $\left[\left(P_{C_{D}}, Y_{C_{D}}\right)\right]$ does not depend of the contravariant derivative $D$.

(iii) If $h$ is a Hermitian metric on $\pi: K \rightarrow M$ and $D$ is a Hermitian contravariant derivative then $P_{C_{D}}$ and $Y_{C_{D}}$ are purely imaginary.

Proof: (i) Let $\{e\}$ be a local basis of $\Gamma(K)$. From (20) and (24) we deduce that there exist a local complex vector field $X_{D}=X_{1}+i X_{2}$ and a local complex function $g_{D}=g_{1}+i g_{2}$ such that

$$
D_{(\alpha, f)} e=\left(\left(\widetilde{X_{D}, g_{D}}\right)(\alpha, f)\right) e
$$

for $(\alpha, f) \in \Omega^{1}(M) \times C^{\infty}(M, \mathrm{R})$, where $\left(\widetilde{X_{D}, g_{D}}\right)=\left(\widetilde{X_{1}, g_{1}}\right)+i\left(\widetilde{X_{2}, g_{2}}\right)$. Thus, using (19), (21), (24), (26) and (27), we have that

$$
\sigma\left(X_{D}, g_{D}\right)=\left(P_{C_{D}}, Y_{C_{D}}\right)
$$

which implies that $\sigma\left(P_{C_{D}}, Y_{C_{D}}\right)=0$.

(ii) Assume that $\bar{D}$ is another contravariant derivative on $\pi: K \rightarrow M$ and that $X_{\bar{D}}$ and $g_{\bar{D}}$ are the corresponding local complex vector field and the corresponding local complex function as in (i). We obtain (see (29))

$$
\left(P_{C_{D}^{-}}, Y_{C_{D}^{-}}\right)=\left(P_{C_{D}}, Y_{C_{D}}\right)+\sigma\left(X_{\bar{D}}^{-}-X_{D}, g_{\bar{D}}^{-} g_{D}\right) .
$$

On the other hand, if we define the mapping

$$
\bar{D}-D:\left(\Omega^{1}(M) \times C^{\infty}(M, \mathbb{R})\right) \times \Gamma(K) \rightarrow \Gamma(K), \quad((\alpha, f), s) \mapsto \bar{D}_{(\alpha, f)} s-D_{(\alpha, f)} s,
$$

then, from (24) and the results of Sec. III, we conclude that there exists a globally defined complex vector field $X_{(\bar{D}-D)}$ and a globally defined complex function $g_{(\bar{D}-D)}$ such that

$$
(\bar{D}-D)((\alpha, f), s)=\left(\left(\overline{\left.X_{(\bar{D}-D)}, g_{(\bar{D}-D)}\right)}\right)(\alpha, f)\right) s .
$$


But in the overlapping we have

$$
X_{(\bar{D}-D)}=X_{\bar{D}}^{-}-X_{D} \quad \text { and } g_{(\bar{D}-D)}=g_{\bar{D}}-g_{D} .
$$

Consequently, by using (30), we prove (ii).

(iii) If $\{e\}$ is a local orthonormal basis of $\Gamma(K)$ then (see (25))

$$
0=h\left(D_{(\alpha, f)} e, e\right)+h\left(e, D_{(\alpha, f)} e\right)=\left(\widetilde{X_{D}, g_{D}}\right)(\alpha, f)+\widetilde{\left(\widetilde{X_{D}, g_{D}}\right)(\alpha, f)},
$$

for all $(\alpha, f) \in \Omega^{1}(M) \times C^{\infty}(M, \mathbb{R})$, where the bar denotes complex conjugation. Taking $f=0$ in (33), we deduce that $\alpha\left(X_{D}\right)+\alpha\left(\bar{X}_{D}\right)=0$ for all $\alpha$. Therefore, $X_{D}$ is purely imaginary which, by (33), implies that $g_{D}$ is also purely imaginary. Since $\left(P_{C_{D}}, Y_{C_{D}}\right)=\sigma\left(X_{D}, g_{D}\right)$, we deduce the result.

Theorem IV.3 suggests us to introduce the following definition.

Definition IV.4: Let $\pi: K \rightarrow M$ be a complex line bundle over a Jacobi manifold $(M, \Lambda, E)$. Suppose that $D$ is a contravariant derivative with curvature $C_{D}$ such that $P_{C_{D}}$ and $Y_{C_{D}}$ are purely imaginary. Then, the cohomology class $\left[\left((i / 2 \pi) P_{C_{D}},(i / 2 \pi) Y_{C_{D}}\right)\right]$ in $H_{L J}^{2}(M)$ is called the real Jacobi-Chern class of the complex line bundle $\pi: K \rightarrow M$ and it is denoted by $j c(K, \Lambda, E)$.

Next, we will show the relation between the usual real Chern class and the real Jacobi-Chern class of a complex line bundle over a Jacobi manifold.

Let $\pi: K \rightarrow M$ be a complex line bundle over a differentiable manifold $M$. If $h$ is a Hermitian metric on $\pi: K \rightarrow M$ and $\boldsymbol{\nabla}$ a Hermitian covariant derivative then there exists a purely imaginary closed 2-form $\Omega_{\nabla}$ such that

$$
\bar{\Omega}_{\nabla}(X, Y)(s)=\Omega_{\nabla}(X, Y)(s)
$$

for all $X, Y \in \mathfrak{X}(M)$ and $s \in \Gamma(K)$, where $\bar{\Omega}_{\nabla}$ is the curvature of the connection $\nabla$, i.e.,

$$
\bar{\Omega}_{\nabla}(X, Y)(s)=\left(\nabla_{X} \circ \nabla_{Y}-\nabla_{Y} \circ \nabla_{X}-\nabla_{[X, Y]}\right)(s) .
$$

The real Chern class $c(K, \mathbb{R}) \in H_{d R}^{2}(M)$ is just the integral cohomology class $\left[(i / 2 \pi) \Omega_{\nabla}\right]$ (see Ref. 1).

Theorem IV.5: Let $\pi: K \rightarrow M$ be a complex line bundle over a Jacobi manifold $(M, \Lambda, E)$. If $c(K, \mathrm{R})$ and $j c(K, \Lambda, E)$ are the real Chern class and the real Jacobi-Chern class, respectively, of $\pi: K \rightarrow M$ then

$$
\widetilde{\#}(c(K, \mathbb{R}))=j c(K, \Lambda, E),
$$

where \#: $H_{d R}^{2}(M) \rightarrow H_{L J}^{2}(M)$ is the induced homomorphism between the de Rham cohomology and the LJ-cohomology.

Proof: If $\boldsymbol{\nabla}$ is a Hermitian covariant derivative and $\{e\}$ is a local basis of $\Gamma(K)$, we have

$$
\nabla_{X} e=\theta(X) e
$$

for all $X \in \mathfrak{X}(M)$, where $\theta$ is the local connection 1 -form. Moreover, if $\Omega=(i / 2 \pi) \Omega_{\nabla}$ we deduce that locally (see Ref. 1)

$$
-2 \pi i \Omega=\Omega_{\nabla}=d \theta
$$

Now, let $D$ be the Hermitian contravariant derivative given by $D_{(\alpha, f)}=\nabla_{(\#, E)(\alpha, f)}$. Denote by $X_{D}$ and $g_{D}$ the local complex vector field and the local complex function which satisfy (28). Using (35), it follows that 


$$
-\widetilde{\#}(\theta)=(-\#(\theta), \theta(E))=\left(X_{D}, g_{D}\right)
$$

Thus, from (29), (36), (37) and Theorem III.2, we conclude that

$$
\widetilde{\#}(\Omega)=\left(\frac{i}{2 \pi} P_{C_{D}}, \frac{i}{2 \pi} Y_{C_{D}}\right)
$$

which proves our result.

Remark IV.6: Let $(M, \Lambda)$ be a Poisson manifold and $\#: H_{d R}^{*}(M) \rightarrow H_{L P}^{*}(M)$ the canonical homomorphism between the de Rham cohomology of $M$ and the LP-cohomology (see Remark III.3). If $\pi: K \rightarrow M$ is a complex line bundle over $M$, Vaisman ${ }^{3}$ defined the Poisson-Chern class $p c(K, \Lambda)$ of $\pi: K \rightarrow M$ as a LP-cohomology class of order 2 so that $\#(c(K, \mathbb{R}))=p c(K, \Lambda)$. Thus, using Remark III.3 and Theorem IV.5, we obtain that

$$
i_{*}(p c(K, \Lambda))=j c(K, \Lambda, 0),
$$

where $j c(K, \Lambda, 0)$ is the Jacobi-Chern class of $\pi: K \rightarrow M$ and $i_{*}: H_{L P}^{*}(M) \rightarrow H_{L J}^{*}(M)$ is the canonical homomorphism between the LP-cohomology and the LJ-cohomology.

\section{PREQUANTIZATION}

Let $(M, \Lambda, E)$ be a Jacobi manifold and $D$ a coutravariant derivative on the complex line bundle $\pi: K \rightarrow M$. Suppose that $x$ is a point of $M$ and that $\omega$ is a 1 -form at $x$. If $K_{x}=\pi^{-1}(x)$ is the fibre over $x$, we define the linear mapping $D_{\omega}: \Gamma(K) \rightarrow K_{x}$ by

$$
D_{\omega}(s)=\left(D_{(\widetilde{\omega}, 0)} s\right)_{x},
$$

where $\widetilde{\omega}$ is a 1 -form on $M$ such that $\widetilde{\omega_{x}}=\omega$. Using (24), we deduce that $D_{\omega}$ does not depend on the extension $\widetilde{\omega}$, i.e., if $\bar{\omega}$ is another 1 -form on $M$ such that $\bar{\omega}_{x}=\omega$ then

$$
\left(D_{(\bar{\omega}, 0)} s\right)_{x}=\left(D_{(\bar{\omega}, 0)} s\right)_{x} .
$$

We will assume that all the contravariant derivatives considered in this section satisfy the following conditions:

(C1) If $E_{x}=0$ then $\left(D_{(0,1)} s\right)_{x}=0$ for all $s \in \Gamma(K)$.

(C2) If $E_{x} \neq 0$ and there exists a 1 -form $\omega$ at $x$ such that $\#_{x}(\omega)=E_{x}$ then

$$
D_{\omega} s-\left(D_{(0,1)} s\right)_{x}=0
$$

for all $s \in \Gamma(K)$.

Note that if $\boldsymbol{\nabla}$ if a usual covariant derivative on $\pi: K \rightarrow M$, then the contravariant derivative $D_{(\alpha, f)}=\nabla_{(\#, E)(a, f)}$ satisfies the above conditions.

Definition V.1: We say that a Jacobi manifold $(M, \Lambda, E)$ is quantizable if there exists a complex line bundle $\pi: K \rightarrow M$ over $M$ such that

$$
\{\widehat{f, g}\}=\hat{f} \circ \hat{g}-\hat{g} \circ \hat{f} \quad f, g \in C^{\infty}(M, \mathbb{R})
$$

with $\hat{f} \in \operatorname{End}_{\mathrm{c}}(\Gamma(K))$ defined by

$$
s \in \Gamma(K) \mapsto \hat{f}(s)=D_{j^{1} f} s+2 \pi i f s=D_{(d f, f)} s+2 \pi i f s,
$$

where $D$ is a contravariant derivative on $\pi: K \rightarrow M$.

For the sake of simplicity and following Refs. 1 and 3, we have forgotten about the Planck constant in the definition of quantizable Jacobi manifold. 
If $C_{D}$ is the curvature of $D$, from (2), (24), (26), (40) and Theorem II.2, we deduce that the condition (39) is equivalent to

$$
C_{D}((d f, f),(d g, g))(s)=-2 \pi i \Lambda(d f, d g) s=-2 \pi i((\widetilde{\Lambda, 0})((d f, f),(d g, g))) s,
$$

for all $s \in \Gamma(K)$. Thus using the fact that $C_{D}$ is trilinear over $C^{\infty}(M, \mathbb{R})$ and its skew-symmetric character we obtain that

$$
C_{D}((\alpha, f),(\beta, g))(s)=-2 \pi i((\widetilde{\Lambda, 0})((\alpha, f),(\beta, g))) s,
$$

for all $(\alpha, f),(\beta, g) \in \Omega^{1}(M) \times C^{\infty}(M, \mathbb{R})$ and $s \in \Gamma(K)$. This implies that (see (27))

$$
\left(\frac{i}{2 \pi} P_{C_{D}}, \frac{i}{2 \pi} Y_{C_{D}}\right)=(\Lambda, 0) \text {. }
$$

Hence $(M, \Lambda, E)$ is quantizable if and only if there is a complex line bundle $\pi: K \rightarrow M$ that possesses a contravariant derivative $D$ satisfying (42). In particular, we must have $\left(P_{C_{D}}, Y_{C_{D}}\right)$ purely imaginary which suggests looking for $K$ together with a Hermitian contravariant derivative. Also, since $\sigma(0,1)=(\Lambda, 0)$ ( $\sigma$ being the LJ-cohomology operator, see $(18)$ ), we deduce that the real Jacobi-Chern class of $\pi: K \rightarrow M$ is null (see Definition IV.4). Moreover, if $\widetilde{\#}: \Omega^{k}(M) \rightarrow \mathscr{V}^{k}(M) \oplus \mathscr{V}^{k-1}(M)$ is the homomorphism of $C^{\infty}(M, \mathrm{R})$-modules given by (23), we obtain:

Theorem V.2: Let $(M, \Lambda, E)$ be a Jacobi manifold. Then, $M$ is quantizable if and only if there exist a vector field $A$, a real differentiable function $f$ and a closed 2-form $\Omega$ which represents an integral cohomology class of $M$ such that

(i) $\quad \#(\Omega)=\sigma(A, f)$.

(ii) If $x$ is a point of $M$ and $E_{x}=0$ then $f(x)=1$.

(iii) If $x$ is a point of $M$ and $\omega$ is a 1 -form at $x$ such that $E_{x} \neq 0$ and $\#_{x}(\omega)=E_{x}$ then $f(x)$ $=\omega\left(A_{x}\right)+1$.

Proof: Suppose that $(M, \Lambda, E)$ is quantizable. Then, there is a complex line bundle $\pi: K \rightarrow M$ over $M$ and a contravariant derivative $D$ on $\pi: K \rightarrow M$ with curvature $C_{D}$ satisfying (42).

Now, let $h$ be a Hermitian metric on $\pi: K \rightarrow M$ and $\nabla$ a Hermitian covariant derivative. Denote by $\bar{\Omega}_{\nabla}$ the curvature of $\nabla$ and by $\Omega_{\nabla}$ the 2 -form given by (34). Then $\Omega=(i / 2 \pi) \Omega_{\nabla}$ is a closed real 2-form which represents an integral cohomology class of $M$. In fact, the real Chern class $c(K, \mathrm{R})$ is just $[\Omega]$ (see Sec. IV).

We consider the contravariant derivative $\bar{D}$ defined by $\bar{D}_{(\alpha, f)}=\nabla_{(\#, E)(\alpha, f)}$. From (18), (38), (42) and Theorem IV. 3, we obtain that there exist a complex vector field $X_{(\bar{D}-D)}$ and a complex function $g_{(\bar{D}-D)}$ such that

$$
\widetilde{\#}(\Omega)=(\Lambda, 0)+\sigma\left(\frac{i}{2 \pi} X_{(\bar{D}-D)}, \frac{i}{2 \pi} g_{(\bar{D}-D)}\right)=\sigma\left(\frac{i}{2 \pi} X_{(\bar{D}-D)}, \frac{i}{2 \pi} g_{(\bar{D}-D)}+1\right) .
$$

Thus we have

$$
\widetilde{\#}(\Omega)=\sigma(A, f),
$$

where $A$ and $f$ are the real vector field and the real function, respectively on $M$ given by

$$
A=\frac{-1}{2 \pi} \operatorname{Im}\left(X_{(\bar{D}-D)}\right), \quad f=\frac{-1}{2 \pi} \operatorname{Im}\left(g_{(\bar{D}-D)}\right)+1
$$


(Here $\operatorname{Im}\left(X_{(\bar{D}-D)}\right.$ and $\operatorname{Im}\left(g_{(\bar{D}-D)}\right)$ denote the imaginary parts of $X_{(\bar{D}-D)}$ and $g_{(\bar{D}-D)}$, respectively). This proves $(i)$. (C2).

(ii) and (iii) follows using (28), (32) and the fact that $D$ and $\bar{D}$ satisfy the conditions (C1) y

Conversely, assume that $A, f$ and $\Omega$ are a vector field, a real function and an integral closed 2-form on $M$ which satisfy the conditions $(i)$, (ii) and (iii). Then, it is well-known (see, for instance, Ref. 1) that there exists a complex line bundle $\pi: K \rightarrow M$ over $M$, endowed with a Hermitian metric $h$, such that $-2 \pi i \Omega$ is the curvature form of a certain Hermitian covariant derivative $\nabla$ on $\pi: K \rightarrow M$.

Define the Hermitian contravariant derivative $D: \Omega^{1}(M) \times C^{\infty}(M, \mathbb{R}) \rightarrow E n d_{\mathrm{C}}(\Gamma(K))$ by

$$
D_{(\alpha, g)} s=\nabla_{(\#, E)(\alpha, g)} s+2 \pi i(\alpha(A)+(f-1) g) s .
$$

A direct computation, using (ii) and (iii), proves that $D$ satisfies the conditions (C1) and (C2). Finally, from (18), (28), (30), (32) and (38), we conclude that

$$
\left(\frac{i}{2 \pi} P_{C_{D}}, \frac{i}{2 \pi} Y_{C_{D}}\right)=(\Lambda, 0) .
$$

Remark V.3: The condition $(i)$ of Theorem V.2 is equivalent to

$$
\#(\Omega)=f A-\mathscr{L}_{A} \Lambda+E \wedge A \quad \text { and }-\#\left(i_{E} \Omega\right)=\#(d f)+\mathscr{L}_{E} A .
$$

Using Theorems III.2 and V.2, we deduce the following

Corollary V.4: Let $(M, \Lambda, E)$ be a Jacobi manifold and $H_{d R}^{*}(M)$ the de Rham cohomology of M. Suppose that $H_{d R}^{2}(M)=\{0\}$. Then, $M$ is quantizable if and only if there exists a 1-cocycle $(A, f) \in \mathfrak{X}(M) \times C^{\infty}(M, \mathbb{R})$ in the LJ-cohomology such that:

(i) If $x$ is a point of $M$ and $E_{x}=0$ we have that $f(x)=1$.

(ii) If $x$ is a point of $M$ and $\omega$ is a 1 -form at $x$ such that $E_{x} \neq 0$ and $\#_{x}(\omega)=E_{x}$ then $f(x)$ $=\omega\left(A_{x}\right)+1$.

\section{EXAMPLES}

Example VI.1: [Poisson, symplectic and Lie-Poisson structures.] Let $(M, \Lambda)$ be a Poisson manifold. Using Theorem V.2, we obtain that $M$ is quantizable as a Jacobi manifold if and only if there exist a vector $A$ and a closed 2-form $\Omega$ that represents an integral cohomology class of $M$ which satisfy (8). Thus, $M$ is quantizable as a Jacobi manifold if and only if $M$ is quantizable as a Poisson manifold in the sense of Vaisman. ${ }^{3}$

In particular, if $M$ is a symplectic manifold with symplectic 2-form $\Phi$, we deduce that $M$ is quantizable if and only if $\Phi$ represents an integral cohomology class of $M$ (see Refs. 1, 2 and 3). On the other hand, since for the Lie-Poisson structure of a coadjoint Lie algebra the Poisson 2-vector is exact in the LP-cohomology, we have that these Poisson structures are always quantizable (see Ref. 3).

Example VI.2: [Jacobi manifolds with l.c.s. characteristic foliation.] Let $(M, \Lambda, E)$ be a Jacobi manifold and suppose that there exists a closed 1-form $\alpha$ such that

$$
E=-\#(\alpha)
$$

and

$$
E_{x}=0, \quad \text { for } x \in M \Rightarrow \alpha_{x}=0 \text {. }
$$


In particular, this implies that $M$ has 1.c.s. characteristic foliation, i.e., all the leaves of the characteristic foliation of $M$ are 1.c.s. manifolds with the induced Jacobi structure (see Sec. II C).

Assume that $M$ is quantizable.

Using (44), (45) and Theorem V.2, we obtain that there exist an integral closed 2-form $\Omega$ and a vector field $A$ such that

$$
\begin{aligned}
& \#(\Omega)=A-\mathscr{L}_{A} \Lambda+E \wedge A-\alpha(A) A, \\
& -\#\left(i_{E} \Omega\right)=-\#(d(\alpha(A)))+[E, A] .
\end{aligned}
$$

From (44), (46) and since $\alpha$ is closed, we deduce that

$$
\#\left(i_{E} \Omega\right)=i_{\alpha}(\#(\Omega))=-E-i_{\alpha}\left(\mathscr{L}_{A} \Lambda\right)=-E-[E, A]+\#\left(\mathscr{L}_{A} \alpha\right)=-E-[E, A]+\#(d(\alpha(A))) .
$$

Thus, using (47), we have that $E=0$, i.e., $M$ is a Poisson manifold. On the other hand, if $M$ is a 1.c.s. manifold with Lee 1 -form $\omega$ then, $\omega$ is closed, $E=-\#(\omega)$ and $E_{x}=0$ if and only if $\omega_{x}$ $=0$ (see Secs. II B and II C). Therefore, we can apply the above results and conclude that a 1.c.s. manifold is quantizable if and only if it is a quantizable symplectic manifold.

Example VI.3: [Jacobi manifolds with contact characteristic foliation.] Let $(M, \Lambda, E)$ be a Jacobi manifold with contact characteristic foliation, i.e., all the leaves of the characteristic foliation are contact manifolds with the induced Jacobi structure. This condition is equivalent to that $E_{x} \notin \operatorname{Im} \#_{x}$, for all $x \in M$ (see Sec. II C). In particular, a contact manifold is trivially a Jacobi manifold with contact characteristic foliation.

We will prove that a Jacobi manifold $M$ with contact characteristic foliation is always quantizable.

For this purpose, we consider the trivial complex line bundle $\pi: K=M \times \mathrm{C} \rightarrow M$. It is clear that, in this case, $\Gamma(K)$ can be identified with the space $C^{\infty}(M, \mathrm{C})$ of $C^{\infty}$ complex-valued functions on $M$. Under this identification, we define the contravariant derivative $D$ by

$$
D_{(\alpha, f)} s=(\#(\alpha)+f E) s-2 \pi i f s,
$$

for all $\alpha \in \Omega^{1}(M), f \in C^{\infty}(M, \mathrm{R})$ and $s \in C^{\infty}(M, \mathrm{C})$. If $h$ is the usual Hermitian metric on $\pi: M$ $\times \mathrm{C} \rightarrow M$ then $D$ is compatible with $h$. Moreover, from (40) and (48), we deduce that

$$
\hat{f}(s)=X_{f}(s) \text {. }
$$

Thus, it is obvious that

$$
\{\widehat{f, g}\}=\hat{f} \circ \hat{g}-\hat{g} \circ \hat{f} .
$$

Notice that the fact that a Jacobi manifold with contact characteristic foliation to be quantizable also follows directly from Theorem V.2.

Remark VI.4: ( $i$ ) If $M$ is a Jacobi manifold with contact characteristic foliation and $\mathfrak{X}_{H}(M)$ is the Lie algebra of the Hamiltonian vector fields, the mapping

$$
C^{\infty}(M, \mathbb{R}) \rightarrow \mathfrak{X}_{H}(M), \quad f \rightarrow X_{f}
$$

is a Lie algebra isomorphism.

(ii) In Ref. 44, Vaisman discussed some basic ideas on geometric quantization under a very general aspect. In that paper, geometric quantization was regarded as a theory of representations of Lie algebras of vector fields on manifolds rather then of Poisson algebras of functions. Complex line bundles and covariant derivatives were used. The symplectic case was studied and some 
classical results were recovered. Moreover, another new situation was also discussed: the case of a contact manifold. In particular, Vaisman proved that a contact manifold is always quantizable and, as in our scheme, a flat complex line bundle was used.

Example VI.5: [Regular Jacobi manifolds.] Let $(\bar{M}, \bar{\Lambda})$ be a quantizable Poisson manifold and $\bar{A}, \bar{\Omega}$ a vector field and an integral closed 2-form on $\bar{M}$ which satisfy (8).

Suppose that $\pi: M \rightarrow \bar{M}$ is the principal circle bundle over $\bar{M}$ corresponding to the 2-form $\bar{\Omega}$, that is, there exists a connection form $\theta$ on $\pi: M \rightarrow \bar{M}$ with curvature form $\bar{\Omega}$. Denote by $(\Lambda, E)$ the associated Jacobi structure on $M$ (see Sec. II D and Ref. 17).

Now, assume that there exists a 1 -form $\bar{\alpha}$ on $\bar{M}$ such that

$$
\bar{A}=\overline{\#}(\bar{\alpha}) .
$$

In such a case, we will prove that $(M, \Lambda, E)$ is a Jacobi manifold with contact characteristic foliation which implies that $M$ is quantizable.

Let $x$ be a point of $M$. We will see that $E_{x} \notin \operatorname{Im} \#_{x}$. Suppose that there exists a 1 -form $\omega$ at $x$ such that $\#_{x}(\omega)=E_{x}$. Then, using (10) and (11), we deduce that

$$
i_{\omega} \bar{\Lambda}_{x}^{H}=0 \quad \text { and } \omega\left(\bar{A}_{x}^{H}\right)=-1 .
$$

On the other hand, from (10), (11) and (50), we obtain that $\bar{A}^{H}=\#\left(\pi^{*} \bar{\alpha}\right)$. Thus $w\left(\bar{A}_{x}^{H}\right)$ $=-\left(\pi^{*} \bar{\alpha}\right)_{x}\left(\#_{x}(w)\right)=-\left(\pi^{*} \bar{\alpha}\right)_{x}\left(E_{x}\right)=0$, which is a contradiction. Two particular cases of the above situation are the following:

(i) If $(\bar{M}, \bar{\Lambda})$ is a quantizable symplectic manifold with symplectic 2-form $\bar{\Phi}$ then $\bar{\Phi}$ represents an integral cohomology class of $\bar{M}$ and we can take $\bar{A}=0$. In this case, the corresponding Jacobi manifold $M$ is a contact manifold (see Ref. 17).

(ii) If $(\bar{M}, \bar{\Phi}, \bar{\eta})$ is a cosymplectic manifold of dimension $2 m+1$ and the closed 2-form $\bar{\Phi}$ represents an integral cohomology class of $\bar{M}$ then, using that $\#(\bar{\Phi})=\bar{\Lambda}$, we have that $\bar{M}$ is quantizable and we also can take $\bar{A}=0$. In this case, the leaves of the characteristic foliation of the Jacobi manifold $M$ are contact manifolds of dimension $2 m+1$ (for more details, see Ref. 17).

If the vector field $\bar{A}$ does not belong to the space $\overline{\#}\left(\Omega^{1}(\bar{M})\right)$ then, in general, the Jacobi manifold $(M, \Lambda, E)$ is not quantizable as the next example proves. Let $(\bar{M}, \bar{\Phi}, \bar{\eta})$ be a cosymplectic manifold with Reeb vector field $\bar{\xi}$. Suppose that the closed 2-form $\bar{\Phi}$ represents an integral cohomology class of $\bar{M}$. As in (ii), $\bar{M}$ is quantizable. But, since $\mathscr{L} \bar{\zeta} \bar{\Lambda}=0$, we can take $\bar{A}=\bar{\xi}$ (instead of $\bar{A}=0$ ). In this case, it is proved in Ref. 17 that $M$ with the Jacobi structure $(\Lambda, E)$ is a l.c.s. manifold. Therefore, $M$ is not quantizable (see Example VI.2).

Example VI.6: [Jacobi manifolds with nonpure characteristic foliation.] We say that a Jacobi manifold has nonpure characteristic foliation $\mathscr{F}$ if there exist leaves of $\mathscr{F}$ of odd and even dimension, i.e., contact and l.c.s. leaves.

Next, we will give four examples of Jacobi manifolds with nonpure characteristic foliation. The first and second examples are not quantizable. However, the two remaining examples are quantizable.

Example VI.6.1: Let $\mathfrak{s b}(2, \mathrm{C})$ be the 3-dimensional real Lie algebra of $2 \times 2$ traceless upper triangular complex matrices with real diagonal elements. A basis $\left\{e_{1}, e_{2}, e_{3}\right\}$ of $\mathfrak{s b}(2, \mathrm{C})$ is given by

$$
e_{1}=\left(\begin{array}{cc}
1 & 0 \\
0 & -1
\end{array}\right), \quad e_{2}=\left(\begin{array}{ll}
0 & 1 \\
0 & 0
\end{array}\right), \quad e_{3}=\left(\begin{array}{ll}
0 & i \\
0 & 0
\end{array}\right)
$$


Suppose that $\mathfrak{s b}(2, \mathrm{C})^{*}$ is the dual space of $\mathfrak{s b}(2, \mathrm{C})$ and that $\left\{e^{1}, e^{2}, e^{3}\right\}$ is the dual basis of $\left\{e_{1}, e_{2}, e_{3}\right\}$. Denote by $(t, q, p)$ the induced global coordinates on $\mathfrak{s} \mathfrak{b}(2, \mathrm{C})^{*} \cong \mathbb{R}^{3}$ by the basis $\left\{e^{1}, e^{2}, e^{3}\right\}$. In these coordinates, the Lie-Poisson structure $\Lambda$ on $\mathfrak{s b}(2, \mathrm{C}) *$ is given by

$$
\Lambda=\frac{\partial}{\partial t} \wedge\left(q \frac{\partial}{\partial q}+p \frac{\partial}{\partial p}\right)
$$

This structure is related to the quantum SU(2) group of Woronowicz (see Ref. 45; see also Refs. $46,47)$. The Poisson manifold $(\mathfrak{s b}(2, \mathrm{C}) *, \Lambda)$ is quantizable (see Example VI.1).

Now, we consider on $\mathfrak{s b}(2, \mathrm{C})^{*}$ the Jacobi structure $(\Lambda, E)$, where $E$ is the vector field

$$
E=\frac{\partial}{\partial t}
$$

The leaves of the characteristic foliation of $\left(\mathfrak{s b}(2, \mathrm{C})^{*}, \Lambda, E\right)$ are the following submanifolds:

(i) 2-dimensional symplectic leaves: the family of open half planes with boundary the line $l: q=0, p=0$.

(ii) 1-dimensional contact leaves: the line $l$.

Hence, all the leaves are quantizable.

On the other hand, in the open subset $U=\mathfrak{s b}(2, \mathrm{C}) *-l$ we have

$$
E=\#(\alpha),
$$

where $\alpha$ is the 1 -form on $U$ defined by

$$
\alpha=\frac{-1}{\left(q^{2}+p^{2}\right)}(q d q+p d p)
$$

Thus $U$ with the induced Jacobi structure is not quantizable (see Example VI.2). Therefore, using Theorem V.2 and Corollary V.4, we conclude that the Jacobi manifold $\left(\mathfrak{s b}(2, \mathrm{C})^{*}, \Lambda, E\right)$ is not quantizable.

Example VI.6.2: Let $\mathfrak{s u}(2, \mathrm{C})$ be the Lie algebra of the special unitary group $\mathrm{SU}(2)$,

$$
\mathfrak{s u}(2, \mathrm{C})=\left\{A \in g l(2, \mathrm{C}) / \overline{A^{t}}=-A, \text { trace } A=0\right\} .
$$

If $\sigma_{1}, \sigma_{2}$ and $\sigma_{3}$ are the Pauli matrices

$$
\sigma_{1}=\left(\begin{array}{ll}
0 & 1 \\
1 & 0
\end{array}\right), \quad \sigma_{2}=\left(\begin{array}{cc}
0 & -i \\
i & 0
\end{array}\right), \quad \sigma_{3}=\left(\begin{array}{cc}
1 & 0 \\
0 & -1
\end{array}\right),
$$

and $e_{j}=-i \sigma_{j}, j=1,2,3$ then $\left\{e_{1}, e_{2}, e_{3}\right\}$ is a basis of $\mathfrak{s u}(2, \mathrm{C})$. Denote by $\left\{e^{1}, e^{2}, e^{3}\right\}$ the dual basis of the dual space $\mathfrak{s u}(2, \mathrm{C})^{*}$ and by $(x, y, z)$ the induced global coordinates on $\mathfrak{s u}(2, \mathrm{C}) * \cong \mathrm{R}^{3}$.

On the product manifold $\mathfrak{s u}(2, \mathrm{C}) * \times \mathrm{R}$, we consider the Jacobi structure $(\Lambda, E)$ given by

$$
\begin{gathered}
\Lambda=z \frac{\partial}{\partial x} \wedge \frac{\partial}{\partial y}+x \frac{\partial}{\partial y} \wedge \frac{\partial}{\partial z}+y \frac{\partial}{\partial z} \wedge \frac{\partial}{\partial x}+t \frac{\partial}{\partial t} \wedge\left(x \frac{\partial}{\partial x}+y \frac{\partial}{\partial y}+z \frac{\partial}{\partial z}\right), \\
E=t \frac{\partial}{\partial t},
\end{gathered}
$$

where $t$ is the usual coordinate on $\mathrm{R}$. 
Notice that $(\Lambda, E)$ induces a Poisson structure on the submanifold $\mathfrak{s u}(2, \mathrm{C})^{*} \times\{0\} \cong \mathfrak{s u}(2, \mathrm{C})^{*}$. This structure is just the usual Lie-Poisson structure on $\mathfrak{s u}(2, \mathrm{C}) *$.

The leaves of the characteristic foliation of the Jacobi manifold $(\mathfrak{s u}(2, \mathrm{C}) * \times \mathbb{R}, \Lambda, E)$ are the following submanifolds:

(i) 4-dimensional g.c.s. leaves: the open subsets

$$
U_{1}=(\mathfrak{s u}(2, \mathrm{C}) *-\{(0,0,0)\}) \times \mathbb{R}^{+}, \quad U_{2}=(\mathfrak{s u}(2, \mathrm{C}) *-\{(0,0,0)\}) \times \mathbb{R}^{-},
$$

$\mathrm{R}^{+}$(respectively, $\mathbb{R}^{-}$) being the space of positive (respectively, negative) real numbers.

(ii) 2-dimensional symplectic leaves: $S^{2}(r) \times\{0\}$, with $r>0$ and $S^{2}(r)$ the 2-dimensional sphere of radius $r$ in $\mathfrak{s u}(2, \mathrm{C})^{*} \cong \mathbb{R}^{3}$.

(iii) 1-dimensional contact leaves: $\{(0,0,0)\} \times \mathrm{R}^{+}$and $\{(0,0,0)\} \times \mathrm{R}^{-}$.

(iv) 0-dimensional symplectic leaves: $\{(0,0,0)\} \times\{0\}$.

The leaves of dimension 4 are not quantizable (see Example VI.2). Moreover, from (51), we deduce that the induced symplectic form on the leaf $S^{2}(r) \times\{0\}$ is integer if and only if $4 \pi r^{3}$ is an integer number. Thus the leaf $S^{2}(r) \times\{0\}$ is quantizable if and only if $4 \pi r^{3}$ is an integer number (see Example VI.1).

Finally, in the open subset $U=(\mathfrak{s u}(2, \mathrm{C}) *-\{(0,0,0)\}) \times \mathbb{R}$ we have that

$$
E=\#\left(-\frac{1}{\left(x^{2}+y^{2}+z^{2}\right)}(x d x+y d y+z d z)\right) \text {. }
$$

Therefore, proceeding as in Example VI.6.1, we conclude that the Jacobi manifold $(\mathfrak{s u}(2, \mathrm{C}) *$ $\times \mathbb{R}, \Lambda, E)$ is not quantizable.

Example VI.6.3: On $\mathbb{R}^{2 m+1}$ we consider the Jacobi structure $(\Lambda, E)$ defined by

$$
\begin{gathered}
\Lambda=\sum_{i=1}^{m}\left(\frac{\partial}{\partial q^{i}}+\left(p_{i} t\right) \frac{\partial}{\partial t}\right) \wedge \frac{\partial}{\partial p_{i}}, \\
E=t \frac{\partial}{\partial t}
\end{gathered}
$$

$\left(t, q^{1}, \ldots, q^{m}, p_{1}, \ldots, p_{m}\right)$ being the usual coordinates on $\mathbb{R}^{2 m+1}$.

The leaves of the characteristic foliation of the Jacobi manifold $\left(\mathrm{R}^{2 m+1}, \Lambda, E\right)$ are the following submanifolds:

$2 m+1$-dimensional contact leaves: the open subsets

$$
U_{1}=\mathrm{R}^{+} \times \mathbb{R}^{2 m}, \quad U_{2}=\mathbb{R}^{-} \times \mathbb{R}^{2 m} .
$$

Notice that the diffeomorphisms

$$
\begin{gathered}
F_{1}: \mathbb{R}^{2 m+1} \rightarrow U_{1}, \quad\left(t, q^{1}, \ldots, q^{m}, p_{1}, \ldots, p_{m}\right) \mapsto\left(e^{t}, q^{1}, \ldots, q^{m}, p_{1}, \ldots, p_{m}\right) \\
F_{2}: \mathbb{R}^{2 m+1} \rightarrow U_{2}, \quad\left(t, q^{1}, \ldots, q^{m}, p_{1}, \ldots, p_{m}\right) \mapsto\left(-e^{t}, q^{1}, \ldots, q^{m}, p_{1}, \ldots, p_{m}\right)
\end{gathered}
$$

are contact transformations, that is, $F_{1}^{*}\left(\eta_{1}\right)=\eta$ and $F_{2}^{*}\left(\eta_{2}\right)=\eta$, where $\eta, \eta_{1}$ and $\eta_{2}$ are the contact 1-forms on $\mathrm{R}^{2 m+1}, U_{1}$ and $U_{2}$, respectively.

(ii) $2 m$-dimensional symplectic leaves: $\{0\} \times \mathbb{R}^{2 m} \cong \mathbb{R}^{2 m}$. We remark that the induced symplectic structure on $\{0\} \times \mathbb{R}^{2 m}$ is just the usual symplectic structure on $\mathbb{R}^{2 m}$.

From the above results, we deduce that the Jacobi structure $(\Lambda, E)$ on $\mathbb{R}^{2 m+1}$ can be considered as an adequate combination of the usual contact structure of $\mathbb{R}^{2 m+1}$ and of the usual symplectic structure of $R^{2 m}$. It is clear that all the leaves are quantizable.

Now, we denote by $\sigma$ the LJ-cohomology operator on $\mathbb{R}^{2 m+1}$ and by $A$ the vector field given by 


$$
A=-\sum_{i=1}^{m} p_{i} \frac{\partial}{\partial p_{i}}
$$

A direct computation, using (52), proves that $\sigma(A, 1)=0$. Therefore, from Corollary V.4, we conclude that the Jacobi manifold $\left(\mathbb{R}^{2 m+1}, \Lambda, E\right)$ is quantizable.

Example VI.6.4: Let $M$ be a differentiable manifold and $E$ a vector field on $M$. We consider on $M$ the Jacobi structure $(\Lambda=0, E)$ (see Sec. II B). The leaves of the characteristic foliation are the maximal integral curves of $E$. Thus if $E_{x}=0$ the leaf $\mathscr{F}_{x}$ over $x$ is the 0 -dimensional symplectic manifold $\mathscr{F}_{x}=\{x\}$ and, if $E_{x} \neq 0$ then $\mathscr{F}_{x}$ is a 1-dimensional contact manifold. Moreover, in this case, $\sigma(0,1)=(0,0)$ (see (18)). Therefore, using Theorem V.2, we have that $(M, \Lambda, E)$ is a quantizable Jacobi manifold.

Remark VI.7: There exist examples of quantizable Poisson manifolds with nonquantizable symplectic leaves. This is the case for the Lie-Poisson structure of a coadjoint Lie algebra (see Ref. 3,27; see also Ref. 48 for a discussion about the relations between the geometric quantization of a Poisson manifold and of its symplectic leaves). Now, in this direction and in our context, an interesting problem is to obtain examples of quantizable Jacobi manifolds with nonpure characteristic foliation and with nonquantizable symplectic leaves.

\section{QUANTIZATION}

The space of sections $\Gamma(K)$ is usually too big for quantization purposes. So, a polarization has to be introduced in order to reduce $\Gamma(K)$ (see, for instance, Refs. 5 and 6). In this section, we will discuss the notion of polarization in the context of Jacobi manifolds and we will see that if $M$ is a quantizable Jacobi manifold and $\mathscr{P}$ is a polarization on $M$ then, using $\mathscr{P}$, we can construct a quantum Hilbert space.

Let $(M, \Lambda, E)$ be a Jacobi manifold and $\{$,$\} the associated Jacobi bracket. If we also denote by$ $\left\{\right.$, \} the natural extension to the space $\left(\Omega^{1}(M) \times C^{\infty}(M, \mathbb{R})\right) \otimes \mathrm{C} \cong\left(\Omega^{1}(M) \otimes \mathrm{C}\right) \times C^{\infty}(M, \mathrm{C})$ of the bracket on $\Omega^{1}(M) \times C^{\infty}(M, \mathrm{R})$ given by (12), we will define a polarization to be a subspace $\mathscr{P}$ of $\Omega^{1}(M) \otimes \mathrm{C}$ such that $\mathscr{P} \times\{0\}$ is a subalgebra of $\left(\left(\Omega^{1}(M) \times C^{\infty}(M, \mathbb{R})\right) \otimes \mathrm{C},\{\},\right)$ and

$$
\Lambda(\alpha, \beta)=0,
$$

for all $\alpha, \beta \in \mathscr{P}$.

We remark that if $\alpha, \beta \in \Omega^{1}(M) \otimes \mathrm{C}$ and $\Lambda(\alpha, \beta)=0$ then $\{(\alpha, 0),(\beta, 0)\} \in\left(\Omega^{1}(M) \otimes \mathrm{C}\right)$ $\times\{0\}$. Usually, one takes a maximal subspace $\mathscr{P}$. But we prefer not to do this here.

Notice that if $f \in C^{\infty}(M, \mathbb{R})$ and $\alpha \in \Omega^{1}(M) \otimes C$ then $\{(d f, f),(\alpha, 0)\} \in\left(\Omega^{1}(M) \otimes \mathrm{C}\right) \times\{0\}$ (see (12)). Thus, we can consider the subalgebra of $\left(C^{\infty}(M, \mathbb{R}),\{\},\right)$ given by

$$
P(\mathscr{P})=\left\{f \in C^{\infty}(M, \mathbb{R}) /\{(d f, f),(\alpha, 0)\} \in \mathscr{P} \times\{0\}, \text { for all } \alpha \in \mathscr{P}\right\} .
$$

$P(\mathscr{P})$ is called the subalgebra of the straightforwardly quantizable observables of $M$. A direct computation, using (12), proves that the above definitions extend those given by Vaisman ${ }^{3}$ for Poisson manifolds.

On the other hand, let $\mathscr{D}$ be the complex line bundle of complex half-densities of a differentiable manifold $M$, which is defined by transition functions that are the square roots of the absolute values of the Jacobians of the coordinate transformations $\widetilde{x}^{i}=\widetilde{x}^{i}\left(x^{j}\right)$, i.e., $\left|\partial x^{j} / \partial \widetilde{x}^{i}\right|^{1 / 2}$. The cross-sections $\varrho$ of $\mathscr{D}$ are called half-densities of $M$ and the Lie derivative $\mathscr{L}$ of such objects can be defined as for tensors. ${ }^{44,49}$

Let $(M, \Lambda, E)$ be a Jacobi manifold which is quantizable. We consider $\pi: K \rightarrow M$ the quantization complex line bundle endowed with a Hermitian metric $h$ and $D$ a quantization Hermitian 
contravariant derivative. We remark that if $M$ is a quantizable Jacobi manifold then we can choose always a Hermitian metric $h$ on $\pi: K \rightarrow M$ and a quantization Hermitian contravariant derivative (see the proof of Theorem V.2).

Using (24) and the properties of $\mathscr{L}$, we can extend the contravariant derivative $D$ to $\Gamma(K$ $\otimes \mathscr{D})$ by

$$
D_{(\alpha, g)}(s \otimes \varrho)=D_{(\alpha, g)} s \otimes \varrho+s \otimes \mathscr{L}_{(\#, E)(\alpha, g)} \varrho,
$$

for all $(\alpha, g) \in\left(\Omega^{1}(M) \times C^{\infty}(M, \mathbb{R})\right) \otimes \mathrm{C}$. Thus for every $f \in C^{\infty}(M, \mathbb{R})$ we can define $\hat{f}: \Gamma(K$ $\otimes \mathscr{D}) \rightarrow \Gamma(K \otimes \mathscr{D})$ by

$$
\hat{f}(s \otimes \varrho)=D_{(d f, f)}(s \otimes \varrho)+2 \pi i f(s \otimes \varrho) .
$$

Notice that if we add (54) to (55) we have

$$
\hat{f}(s \otimes \varrho)=\hat{f}(s) \otimes \varrho+s \otimes \mathscr{L}_{X_{f}} \varrho,
$$

for all $f \in C^{\infty}(M, \mathbb{R})$ and $s \otimes \varrho \in \Gamma(K \otimes \mathscr{D})$.

Using (7), (56) and the properties of the Lie derivative we obtain that

$$
\{\widehat{f, g}\}(s \otimes \varrho)=(\hat{f} \circ \hat{g}-\hat{g} \circ \hat{f})(s \otimes \varrho) .
$$

On the other hand, from (26), (40), (41), (54), (56) and Theorem II.2 we deduce that

$$
D_{(\alpha, g)} \hat{f}(s \otimes \varrho)=\hat{f} D_{(\alpha, g)}(s \otimes \varrho)-D_{\{(d f, f),(\alpha, g)\}}(s \otimes \varrho)+2 \pi i g E(f)(s \otimes \varrho),
$$

for all $(\alpha, g) \in\left(\Omega^{1}(M) \times C^{\infty}(M, \mathrm{R})\right) \otimes \mathrm{C}, f \in C^{\infty}(M, \mathrm{R})$ and $s \otimes \varrho \in \Gamma(K \otimes \mathscr{D})$. Set

$$
\mathscr{H}_{0}=\left\{v \in \Gamma(K \otimes \mathscr{D}) / D_{(\alpha, 0)} v=0 \text {, for all } \alpha \in \mathscr{P}\right\} .
$$

If $f \in P(\mathscr{P})$ and $v \in \mathscr{H}_{0}$ then, using (57), we obtain that $\hat{f}(v) \in \mathscr{H}_{0}$. Thus $\hat{f}_{\mid \mathscr{C}_{0}}: \mathscr{H}_{0} \rightarrow \mathscr{H}_{0}$ is well defined. Hence we can use $\mathscr{H}_{0}$ as a quantization space for $P(\mathscr{P})$.

A difficulty of this scheme is that it is unclear that $\mathscr{H}_{0} \neq 0$ (a Bohr-Sommerfeld condition). In what follows, we will assume that this condition holds. Now, if $M$ is compact then $\mathscr{H}_{0}$ can be made a pre-Hilbert space with the scalar product

$$
\left\langle s_{1} \otimes \varrho_{1}, s_{2} \otimes \varrho_{2}\right\rangle=\int_{M} h\left(s_{1}, s_{2}\right) \varrho_{1} \bar{\varrho}_{2},
$$

where the bar denotes complex conjugation.

Moreover, using (25), (40), (56) and (59) and the density version of Stokes' theorem ${ }^{44,49}$ we obtain that the operations $\hat{f}$ defined in (55) are anti-Hermitian, i.e.,

$$
\left\langle\hat{f}\left(s_{1} \otimes \varrho_{1}\right), s_{2} \otimes \varrho_{2}\right\rangle+\left\langle s_{1} \otimes \varrho_{1}, \hat{f}\left(s_{2} \otimes \varrho_{2}\right)\right\rangle=\int_{M} \mathscr{L}_{X_{f}}\left(h\left(s_{1}, s_{2}\right) \varrho_{1} \bar{\varrho}_{2}\right)=0 .
$$

If we get the operators $i \hat{f}$ then we obtain Hermitian operators. Finally, if we want a Hilbert space, we will just take the completation of $\mathscr{H}_{0}$.

Remark VII.1: If $(M, \Lambda)$ is a Poisson manifold a direct computation, using Definition V.1, proves that the mapping

$$
\widetilde{D}: \Omega^{1}(M) \rightarrow \operatorname{End}_{\mathrm{C}}(\Gamma(K)), \quad \alpha \in \Omega^{1}(M) \mapsto \widetilde{D}_{\alpha}=D_{(\alpha, 0)}
$$


is a quantization Hermitian contravariant derivative in the sense of Vaisman ${ }^{3}$ (notice that in this case, from the condition $(\mathbf{C 1})$, we have that $D_{(0,1)} s=0$, for all $\left.s \in \Gamma(K)\right)$. Furthermore,

$$
\mathscr{H}_{0}=\left\{v \in \Gamma(T \otimes \mathscr{D}) / \grave{D}_{\alpha} v=0 \text {, for all } \alpha \in \mathscr{P}\right\} .
$$

Thus, the above construction of the pre-Hilbert space $\left(\mathscr{H}_{0},\langle\rangle,\right)$ for compact quantizable Jacobi manifolds extends the one given by Vaisman for compact quantizable Poisson manifolds (see Ref. 3).

If the Jacobi manifold $M$ is not compact, we will postulate a special regularity condition. Let $\mathscr{P}_{0}$ be the subspace of $\Omega^{1}(M)$ defined by $\mathscr{P}_{0}=\mathscr{P} \cap \Omega^{1}(M)$. The complexification of $\mathscr{P}_{0}$ is $\mathscr{P} \cap \bar{P}$ and $\mathscr{P}_{0} \times\{0\}$ is a subalgebra of $\left(\Omega^{1}(M) \times C^{\infty}(M, \mathbb{R}),\{\},\right)$. We also have that

$$
\Lambda(\alpha, \beta)=0,
$$

for all $\alpha, \beta \in \mathscr{P}_{0}$. Moreover, we will assume that $\#\left(\mathscr{P}_{0}\right)$ defines a regular foliation $\mathscr{F}$ of $M$ which fibers $M$ over a Hausdorff manifold $N=M / \mathscr{F}$. Notice that, in such a case, if $f \in P(\mathscr{P})$ then, from (53) and Theorem II.2, we deduce that the Hamiltonian vector field $X_{f}$ projects onto a vector field $\widetilde{X}_{f}$ on $N$ and we have

$$
\hat{f}\left(s \otimes \tau^{*} \widetilde{\varrho}\right)=\hat{f}(s) \otimes \tau^{*} \widetilde{\varrho}+s \otimes \mathscr{D}_{X_{f}}\left(\tau^{*} \widetilde{\varrho}\right)=\hat{f}(s) \otimes \tau^{*} \widetilde{\varrho}+s \otimes \tau^{*}\left(\mathscr{L}_{\bar{X}_{f}} \widetilde{\varrho}\right),
$$

for all $s \in \Gamma(K)$ and $\widetilde{\varrho}$ a complex half-density of $N$, where $\tau: M \rightarrow N=M / \mathscr{F}$ is the canonical projection. Thus, for the definition of $\mathscr{H}_{0}$, instead of using half-densities of $M$ we will use $\mathscr{F}$-transversal half-densities $\varrho$ obtained by lifting half-densities of $N$ to $M$. It is clear that if $\alpha$ $\in \mathscr{P}_{0}$, we have that $\mathscr{L}_{\#(\alpha)}\left(\tau^{*} \widetilde{\varrho}\right)=0$. Using this fact, (54) and (58), we can prove that if $s_{i}$ $\otimes \tau^{*} \widetilde{\varrho}_{i} \in \mathscr{H}_{0}, i=1,2$, then

$$
h\left(s_{1}, s_{2}\right)\left(\tau^{*} \widetilde{\varrho_{1}}\right)\left(\tau^{*} \overline{\widetilde{\varrho}_{2}}\right)
$$

projects to a complex 1-density of $N$. Generally, one may expect to have a nonzero subspace $\mathscr{H}_{0}^{c}$ of $\mathscr{H}_{0}$, such that for all $v \in \mathscr{H}_{0}^{c}$, its support projects onto a compact subset of $N$. Then (59) with $M$ replaced by $N$ yields a pre-Hilbert structure on $\mathscr{H}_{0}^{c}$. Furthermore, if $f \in P(\mathscr{P})$ and $\hat{f}$ is the corresponding operator (see (60)) then, proceeding as in the case when $M$ is compact, we conclude that

$$
\left\langle\hat{f}\left(s_{1} \otimes \tau^{*} \widetilde{\varrho}_{1}\right), s_{2} \otimes \tau^{*} \widetilde{\varrho}_{2}\right\rangle+\left\langle s_{1} \otimes \tau^{*} \widetilde{\varrho}_{1}, \hat{f}\left(s_{2} \otimes \tau^{*} \widetilde{\varrho}_{2}\right)\right\rangle=\int_{N} \mathscr{L}_{X_{f}} \widetilde{\varrho}=0,
$$

where $\widetilde{\varrho}$ is the complex 1-density of $N$ on which projects $h\left(s_{1}, s_{2}\right)\left(\tau^{*} \widetilde{\varrho_{1}}\right)\left(\tau^{*} \widetilde{\varrho_{2}}\right)$.

Remark VII.2: The above construction extends the one given by Vaisman for Poisson manifolds. ${ }^{3}$

Next, we will study the quantization of some examples of Jacobi manifolds.

Example VII.3: Let $\theta$ be the usual contact structure on $M=\mathbb{R}^{2 m+1} \cong \mathbb{R} \times \mathrm{C}^{m}$ given by

$$
\theta=d t-\sum_{j} p_{j} d q^{j}=d t+\frac{i}{4} \sum_{j}\left(z_{j}-\overline{z_{j}}\right)\left(d z_{j}+d \overline{z_{j}}\right)
$$

where $\left(t, q^{1}, \ldots, q^{m}, p_{1}, \ldots, p_{m}\right)$ are the usual coordinates on $\mathbb{R}^{2 m+1}, z_{j}=q^{j}+i p_{j}$ and $\overline{z_{j}}=q^{j}$ $-i p_{j}$. Then the associated Jacobi structure $(\Lambda, E)$ is defined by

$$
\Lambda=\sum_{j}\left(\frac{\partial}{\partial q^{j}}+p_{j} \frac{\partial}{\partial t}\right) \wedge \frac{\partial}{\partial p_{j}}=-2 i \sum_{j} \frac{\partial}{\partial z_{j}} \Lambda \frac{\partial}{\partial \bar{z}_{j}}+\frac{1}{2} \sum_{j}\left(z_{j}-\bar{z}_{j}\right) \frac{\partial}{\partial t} \wedge\left(\frac{\partial}{\partial z_{j}}-\frac{\partial}{\partial \bar{z}_{j}}\right),
$$




$$
E=\frac{\partial}{\partial t} \text {. }
$$

We have proved in Example VI.3 that $(M, \Lambda, E)$ is a quantizable Jacobi manifold and that the trivial complex line bundle $\pi: K=M \times \mathrm{C} \rightarrow M$ is the quantum bundle.

A convenient complex polarization is $\mathscr{P}_{1}=\operatorname{span}\left\{d z_{j}\right\}_{j=1}^{m}$, and then we see that $P\left(\mathscr{P}_{1}\right)$ consists of the functions $f \in C^{\infty}(M, \mathbb{R})$ such that

$$
\left(\#\left(d z_{j}\right)\right)(f)=h_{j}^{f \circ} \mathrm{pr}_{2},
$$

for $j \in\{1, \ldots, m\}$, where $p r_{2}: M=\mathrm{R} \times \mathrm{C}^{m} \rightarrow \mathrm{C}^{m}$ is the canonical projection onto the second factor and $h_{j}^{f}: \mathrm{C}^{m} \rightarrow \mathrm{C}$ is a complex analytic function on $\mathrm{C}^{m}$. Notice that $\mathscr{P}_{1} \cap \overline{\mathscr{P}}_{1}=\{0\}$.

On the other hand, the bundle $\mathscr{D}$ of complex half-densities over $M$ is also trivial and it has a basis that can be written formally as

$$
\gamma=|\beta|^{1 / 2}
$$

where

$$
\beta=d t \wedge d q^{1} \wedge \cdots \wedge d q^{m} \wedge d p_{1} \wedge \cdots \wedge d p_{m}=\left(\frac{i}{2}\right)^{m} d t \wedge d z_{1} \wedge \cdots \wedge d z_{m} \wedge d \overline{z_{1}} \wedge \cdots \wedge d \overline{z_{m}} .
$$

Then, if $v \in \Gamma(K \otimes \mathscr{D}), v$ can be seen as $v=1 \otimes \varphi \gamma$, where $\varphi$ is a complex-valued function on $M$.

Now, if we denote by $D$ the Hermitian contravariant derivative given by (48), $D$ can be extended to $\Gamma(K \otimes \mathscr{D})$ as in (54). Then, we obtain

$$
D_{\left(d z_{j}, 0\right)}(1 \otimes \varphi \gamma)=1 \otimes \mathscr{L}_{\#\left(d z_{j}\right)}(\varphi \gamma) .
$$

Therefore, using the fact that $\mathscr{L}_{X} \gamma=\left(\frac{1}{2} \operatorname{div} X\right) \gamma$ (see, for instance, Ref. 44), it follows that

$$
D_{\left(d z_{j}, 0\right)}(1 \otimes \varphi \gamma)=0
$$

if and only if

$$
\left(\#\left(d z_{j}\right)\right)(\varphi)=-2 i \frac{\partial \varphi}{\partial \bar{z}_{j}}-\frac{1}{2}\left(z_{j}-\overline{z_{j}}\right) \frac{\partial \varphi}{\partial t}=0 .
$$

Hence the quantization space $\mathscr{H}_{0}$ can be identified with the space

$$
\mathscr{H}_{0}=\left\{\varphi \in C^{\infty}(M, \mathrm{C}) / 2 i \frac{\partial \varphi}{\partial \bar{z}_{j}}+\frac{1}{2}\left(z_{j}-\overline{z_{j}}\right) \frac{\partial \varphi}{\partial t}=0\right\} .
$$

On the other hand, if $f \in P\left(\mathscr{P}_{1}\right)$ and $\varphi \in \mathscr{H}_{0}$ then, using (49) and (56), we deduce that

$$
\hat{f}(\varphi)=X_{f}(\varphi)+\frac{1}{2} \varphi\left(\operatorname{div} X_{f}\right) .
$$

Moreover, the scalar product of two functions $\varphi_{1}, \varphi_{2} \in \mathscr{H}_{0}$ with compact support is

$$
\left\langle\varphi_{1}, \varphi_{2}\right\rangle=\int_{M} \varphi_{1} \bar{\varphi}_{2} \beta
$$

Now, we consider the real polarization $\mathscr{P}_{2}=\operatorname{span}\left\{d q^{j}\right\}_{j=1}^{m}$. 
In this case, a $C^{\infty}$ real-valued function $f$ belongs to the subalgebra $P\left(\mathscr{P}_{2}\right)$ if and only if $f$ is an affine function in the coordinates $p_{j}$ of the form

$$
f=\sum_{j=1}^{m} f^{j}\left(q^{1}, \ldots, q^{m}\right) p_{j}+h\left(t, q^{1}, \ldots, q^{m}\right)
$$

with $f^{j} \in C^{\infty}\left(\mathbb{R}^{m}, \mathbb{R}\right)$ and $h \in C^{\infty}\left(\mathbb{R}^{m+1}, \mathbb{R}\right)$.

We have that $\left(\mathscr{P}_{2}\right)_{0}=\mathscr{P}_{2} \cap \Omega^{1}(M)=\mathscr{P}_{2}$ and that $\#\left(\mathscr{P}_{2}\right)_{0}$ defines a regular foliation $\mathscr{F}$ of $M$ generated by the vector fields $\left\{\partial / \partial p_{j}\right\}_{j=1}^{m}$.

Moreover, the space of leaves $N=M / \mathscr{F}$ can be identified with $\mathbb{R}^{m+1}$ and under this identification the canonical projection $\tau: M \rightarrow M / \mathscr{F} \cong \mathrm{R}^{m+1}$ is the mapping given by

$$
\tau\left(t, q^{1}, q^{2}, \ldots, q^{m}, p_{1}, \ldots, p_{m}\right)=\left(t, q^{1}, \ldots, q^{m}\right) .
$$

If we denote by $\widetilde{\mathscr{D}}$ the bundle of half-densities over $N$ and by $\widetilde{\gamma}$ the basis of this bundle, then we obtain

$$
D_{\left(d q^{j}, 0\right)}\left(1 \otimes \varphi \tau^{*}(\widetilde{\gamma})\right)=\left(\#\left(d q^{j}\right)\right)(\varphi)\left(1 \otimes \tau^{*}(\widetilde{\gamma})\right)
$$

where $D$ is the extension of the contravariant derivative defined in (54) to the sections of the form $1 \otimes \varphi \tau^{*}(\widetilde{\gamma}), \varphi$ being a complex-valued function on $M$.

Using (61) we conclude that $1 \otimes \varphi \tau^{*}(\widetilde{\gamma}) \in \mathscr{H}_{0}$ if and only if $\partial \varphi / \partial p_{j}=0$. Hence $\mathscr{H}_{0}^{c}$ can be identified with the space of $C^{\infty}$ complex-valued functions on $\mathbb{R}^{m+1}$ with compact support. Furthermore, the scalar product of two functions $\varphi_{1}, \varphi_{2} \in \mathscr{H}_{0}^{c}$ is given by

$$
\left\langle\varphi_{1}, \varphi_{2}\right\rangle=\int_{\mathbb{R}^{m+1}} \varphi_{1} \bar{\varphi}_{2} d t d q^{1} \cdots d q^{m} .
$$

Example VII.4: Let $(\Lambda, E)$ be the quantizable Jacobi structure on $M=\mathbb{R}^{2 m+1}$ given by (52) (see Example VI.6.3). Using the identification $\mathbb{R}^{2 m+1} \cong \mathbb{R} \times \mathbb{C}^{m}$ we have

$$
\Lambda=-2 i \sum_{j} \frac{\partial}{\partial z_{j}} \wedge \frac{\partial}{\partial \overline{z_{j}}}+\frac{t}{2} \sum_{j}\left(z_{j}-\overline{z_{j}}\right) \frac{\partial}{\partial t} \wedge\left(\frac{\partial}{\partial z_{j}}-\frac{\partial}{\partial \overline{z_{j}}}\right),
$$

where $\left(t, q^{1}, \ldots, q^{m}, p_{1}, \ldots, p_{m}\right)$ are the usual coordinates on $\mathbb{R}^{2 m+1}, z_{j}=q^{j}+i p_{j}$ and $\overline{z_{j}}=q^{j}$ $-i p_{j}$.

Clearly, the quantum bundle is the trivial complex line bundle $\pi: K=M \times \mathrm{C} \rightarrow M$ and the quantization contravariant derivative $D$ on $\pi: M \times \mathrm{C} \rightarrow M$ is defined by (see (43))

$$
D_{(\alpha, g)} s=(\#(\alpha)+g E)(s)+2 \pi i \alpha(A),
$$

where $A$ is the vector field

$$
A=-\sum_{j} p_{j} \frac{\partial}{\partial p_{j}}=-\frac{1}{2} \sum_{j}\left(z_{j}-\overline{z_{j}}\right)\left(\frac{\partial}{\partial z_{j}}-\frac{\partial}{\partial \overline{z_{j}}}\right) .
$$

Now, we consider the polarization $\mathscr{P}=\operatorname{span}\left\{d z_{j}\right\}_{j=1}^{m}$. Then, $P(\mathscr{P})$ consists of the functions $f$ $\in C^{\infty}(M, \mathbb{R})$ such that

$$
\left(\#\left(d z_{j}\right)\right)(f)=h_{j}^{f} \text { op } r_{2}
$$

for $j \in\{1, \ldots, m\}$, where $p r_{2}: M=\mathrm{R} \times \mathrm{C}^{m} \rightarrow \mathrm{C}^{m}$ is the canonical projection onto the second factor and $h_{j}^{f}: \mathrm{C}^{m} \rightarrow \mathrm{C}$ is a complex analytic function on $\mathbb{C}^{m}$. 
Proceeding as in the above example, the sections of the complex line bundle $K \otimes \mathscr{D}$ can be seen as $v=1 \otimes \varphi \gamma$, where $\varphi$ is a complex-valued function on $M$ and $\gamma$ is a basis of the bundle of half-densities $\mathscr{D}$ over $M$.

Since

$$
D_{\left(d z_{j}, 0\right)}(1 \otimes \varphi \gamma)=\left(\left(\#\left(d z_{j}\right)\right)(\varphi)-\varphi\left(z_{j}-\overline{z_{j}}\right)\left(i \pi+\frac{1}{4}\right)\right)(1 \otimes \gamma),
$$

the space $\mathscr{H}_{0}$ can be identified with

$$
\mathscr{H}_{0}=\left\{\varphi \in C^{\infty}(M, \mathrm{C}) / 2 i \frac{\partial \varphi}{\partial \bar{z}_{j}}+\left(z_{j}-\bar{z}_{j}\right)\left(\varphi\left(i \pi+\frac{1}{4}\right)+\frac{t}{2} \frac{\partial \varphi}{\partial t}\right)=0\right\} .
$$

Then, if $f \in P(\mathscr{P})$ and $\varphi \in \mathscr{H}_{0}$, the quantum operator $\hat{f}$ is defined by

$$
\hat{f}(\varphi)=\varphi\left(2 \pi i A(f)+2 \pi i f+\frac{1}{2} d i v X_{f}\right)+X_{f}(\varphi)
$$

and the scalar product of two functions $\varphi_{1}, \varphi_{2} \in \mathscr{H}_{0}$ with compact support is

$$
\left\langle\varphi_{1}, \varphi_{2}\right\rangle=\int_{M} \varphi_{1} \bar{\varphi}_{2} d t d q^{1} \cdots d q^{m} d p_{1} \cdots d p_{m}
$$

As in the above example, we also can consider the real polarization $\mathscr{P}=\operatorname{span}\left\{d q^{j}\right\}_{j=1}^{m}$ and obtain similar results.

Remark VII.5: (i) A complex foliation $\mathscr{F}$ on a $(2 m+1)$-dimensional contact manifold $(M, \theta)$ is said to be Sasakian if $\mathscr{F} \cap \overline{\mathscr{F}}=\{0\},\langle\mathscr{F}, \theta\rangle=0$ and $\operatorname{dim}_{\mathrm{C}} \mathscr{F}=m$. There is a close relation between Sasakian foliations and a particular class of contact manifolds, the Sasakian manifolds (for the definition and properties of Sasakian manifolds we refer to Ref. 34). This relation justifies the name of Sasakian foliation. In fact, it can be proved ${ }^{50}$ that a Sasakian manifold admits a Sasakian foliation. The Sasakian foliations play the same role in contact geometry that the Kähler polarizations in symplectic geometry. We remark that in Example VII.3, $\mathscr{F}_{1}=\#\left(\mathscr{P}_{1}\right)$ is a Sasakian foliation.

(ii) A real foliation $\mathscr{F}$ on a $(2 m+1)$-dimensional contact manifold $(M, \theta)$ is said to be Legendre if $\langle\mathscr{F}, \theta\rangle=0$ and $\operatorname{dim}_{\mathrm{R}} \mathscr{F}=m$ or equivalently, if the leaves of $\mathscr{F}$ are Legendre submanifolds of $M$ (see Refs. 31,34,51). The Legendre foliations play the same role in contact geometry that the Lagrangian foliations in symplectic geometry. We remark that in Example VII.3, $\mathscr{F}_{2}$ $=\#\left(\mathscr{P}_{2}\right)$ is a Legendre foliation.

(iii) In a next paper ${ }^{50}$ we will discuss the geometric quantization of a contact manifold on which there exists a Sasakian or Legendre foliation.

\section{PREQUANTIZATION REPRESENTATIONS}

In Ref. 3 (see also Ref. 27), Vaisman have extended to Jacobi manifolds Urwin's definition of (1-differentiable) prequantization representations for complex line bundles over symplectic manifolds (see Ref. 23). Vaisman has discussed the existence of such representations using covariant derivatives.

In this section, we will characterize the existence of (1-differentiable) prequantization representations for Jacobi manifolds in terms of contravariant derivatives and of the real Jacobi-Chern class. We recall the definition of prequantization representation (see Refs. 3,27).

Let $(M, \Lambda, E)$ be a Jacobi manifold with Jacobi bracket $\{$,$\} and \pi: K \rightarrow M$ a complex line bundle over $M$. A prequantization representation of $\left(C^{\infty}(M, \mathbb{R}),\{\},\right)$ is a Lie algebra homomor- 
phism $Q$ of the algebra $\left(C^{\infty}(M, \mathbb{R}),\{\},\right)$ into the algebra of the first order differential operators on $\Gamma(K)$ with the usual commutator as product such that for all $f \in C^{\infty}(M, \mathbb{R})$ one has that the symbol of $Q(f)$ is $X_{f}$.

Remember that an operator $Q(f)$ as requested is one which acts on $s \in \Gamma(K)$ as follows: if $e$ is a local basis of cross sections of $K$ then $s=g e$ for a complex valued funtion $g$ and $Q(f)(s)$ $=(X(g)+\varphi g) e$, where $X$ is a vector field and $\varphi$ is a function. The vector field $X$ does not change if $e$ is changed to $\widetilde{e}=u e$ hence, $X$ is global on $M$, and it is called the symbol of $Q$. If $\pi: K \rightarrow M$ is a complex line bundle over a Jacobi manifold $(M, \Lambda, E), h$ is a Hermitian metric on $\pi: K \rightarrow M$ and $D$ is a Hermitian contravariant derivative, it follows that the symbol of $D_{(d f, f)}$ is exactly $X_{f}$ (see (24)). Thus, if $Q$ is a prequantization representation, $Q$ must be of the form

$$
Q(f)(s)=D_{j^{1} f} s+2 \pi i m(f) s=D_{(d f, f)} s+2 \pi i m(f) s,
$$

where $m(f)$ is given by a R-linear mapping $m: C^{\infty}(M, \mathbb{R}) \rightarrow C^{\infty}(M, \mathrm{C})$. Since $Q$ is a Lie algebra homomorphism then

$$
Q(\{f, g\})=\left[Q_{f}, Q_{g}\right]
$$

[,] being the usual commutator on the first order differential operators on $\Gamma(K)$.

This commutation condition, using (15), (24), (26), (62) and Theorem II.2, becomes

$$
\frac{i}{2 \pi} C_{D}((d f, f),(d g, g))(s)=X_{f}(m(g)) s-X_{g}(m(f)) s-m(\{f, g\}) s=\left(\partial_{H} m\right)(f, g) s,
$$

where $C_{D}$ is the curvature of $D$ and $\partial_{H}$ is the H-Chevalley-Eilenberg cohomology operator. Since the right-hand of (63) is real ( $D$ is a Hermitian contravariant derivative), the imaginary part of $m$ is a $\mathrm{H}$-Chevalley-Eilenberg 1-cocycle. Thus, if $m=m_{1}+i m_{2}$ and $j_{2}: \mathscr{V}^{2}(M) \oplus \mathscr{V}^{1}(M)$ $\rightarrow C_{H C E}^{2}(M)$ is the monomorphism given by (16), we have that (see (20) and (27))

$$
\partial_{H} m_{1}=j_{2}\left(\frac{i}{2 \pi} P_{C_{D}}, \frac{i}{2 \pi} Y_{C_{D}}\right), \quad \partial_{H} m_{2}=0 .
$$

Therefore, modulo 1-cocycles in the H-Chevalley-Eilenberg cohomology, it suffices to find one real solution $m=m_{1}$.

The prequantization representation $Q$ is said to be 1-differentiable if the mapping $m_{1}: C^{\infty}(M, \mathbb{R}) \rightarrow C^{\infty}(M, \mathbb{R})$ is a 1-differentiable $\mathrm{H}$-Chevalley-Eilenberg 1-cochain, that is, there exists a vector field $A$ on $M$ and a function $\varphi$ such that

$$
m_{1}(f)=j_{2}(A, \varphi)(f)=A(f)+\varphi f
$$

for all $f \in C^{\infty}(M, \mathbb{R})$.

Notice that, from (31), we deduce that this definition only depends of the prequantization representation $Q$, i.e., it does not depend of the Hermitian contravariant derivative $D$.

Denote by $j_{*}: H_{L J}^{2}(M) \rightarrow H_{H C E}^{2}(M)$ the induced homomorphism in cohomology by $j_{2}: \mathscr{V}^{2}(M) \oplus \mathscr{V}^{1}(M) \rightarrow C_{H C E}^{2}(M)$. Then, in the following theorem, we characterize the existence of (1-differentiable) prequantization representations.

Theorem VIII.1: Let $(M, \Lambda, E)$ be a Jacobi manifold. A complex line bundle $\pi: K \rightarrow M$ has prequantization representations if and only if

$$
j_{*}(j c(K, \Lambda, E))=0,
$$


where $j_{*}$ is the homomorphism defined above and $j c(K, \Lambda, E)$ is the real Jacobi-Chern class. Moreover, $K$ admits 1-differentiable prequantization representations if and only if $j c(K, \Lambda, E)$ $=0$.

Proof: The first part of this theorem follows from (64) and Definition IV.4. Now, if we have a 1-differentiable prequantization representation then, using (17), (64) and (65), we obtain that there exist $A \in \mathfrak{X}(M)$ and $\varphi \in C^{\infty}(M, \mathrm{R})$ such that

$$
\left(\frac{i}{2 \pi} P_{C_{D}}, \frac{i}{2 \pi} Y_{C_{D}}\right)=\sigma(A, \varphi)
$$

where $D$ is a Hermitian contravariant derivative and $\sigma$ is the LJ-cohomology operator. Hence $j c(K, \Lambda, E)=0$.

Conversely, if $j c(K, \Lambda, E)=0$ and $D$ is a Hermitian contravariant derivative, then there exists $(A, \varphi) \in \mathfrak{X}(M) \times C^{\infty}(M, \mathbb{R}) \quad$ satisfying $\quad$ (66). Define $m=m_{1}: C^{\infty}(M, \mathbb{R}) \rightarrow C^{\infty}(M, \mathbb{R})$ by $m$ $=j_{2}(A, \varphi)$. From $(66)$, we deduce that

$$
Q(f)(s)=D_{(d f, f)} s+2 \pi i m(f) s
$$

is a 1-differentiable prequantization representation.

Remark VIII.2: Let $(M, \Lambda)$ be a Poisson manifold. In this case, the H-Chevalley-Eilenberg cohomology of $M$ is just the Chevalley-Eilenberg cohomology and we have a homomorphism $l_{*}: H_{L P}^{*}(M) \rightarrow H_{C E}^{*}(M)$ between the LP-cohomology and the Chevalley-Eilenberg cohomology. In fact, $l_{*}=j_{*} \circ i_{*}$ where $i_{*}: H_{L P}^{*}(M) \rightarrow H_{L J}^{*}(M)$ is the canonical homomorphism between the LP-cohomology and the LJ-cohomology (see Remark III.3). Thus, using Remark IV.6 and Theorem VIII.1, we deduce that a complex line bundle $\pi: K \rightarrow M$ has prequantization representations if and only if $l_{*}(p c(K, \Lambda))=0, p c(K, \Lambda)$ being the Poisson-Chern class of $\pi: K \rightarrow M$. Moreover, $K$ admits 1-differentiable prequantization representations if and only if $i_{*}(p c(K, \Lambda))=j c(K, \Lambda, 0)$ $=0$, that is (see (18)), if $p c(K, \Lambda)=[f \Lambda]$ with $f$ a Casimir function, i.e., $\#(d f)=0$. This result is just the one obtained by Vaisman in Ref. 3 (see also Ref. 27).

\section{ACKNOWLEDGMENTS}

This work has been partially supported through grants DGICYT (Spain) (Project No. PB940106) and University of La Laguna (Spain). M.L. wishes to express his gratitude for the hospitality offered to him in the Departamento de Matemática Fundamental (University of La Laguna) where part of this work was conceived. We also acknowledge Professor Ch. M. Marle for calling our attention to the note by Y. Kerbrat and Z. Souici-Benhammadi.

\footnotetext{
${ }^{1}$ B. Kostant, "Quantization and unitary representations," in Lectures in Modern Analysis and Applications III, edited by C. T. Taam, Lecture Notes in Math. 170 (Springer, Berlin, 1970), pp. 87-207.

${ }^{2}$ J. M. Souriau, Structures des Systèmes Dynamiques (Dunod, Paris, 1969).

${ }^{3}$ I. Vaisman, "On the geometric quantization of Poisson manifolds," J. Math. Phys. 32, 3339-3345 (1991).

${ }^{4}$ J. Huebschmann, "Poisson cohomology and quantization,"' J. Reine Angew. Math. 408, 57-113 (1990).

${ }^{5}$ J. Sniatycki, Geometric Quantization and Quantum Mechanics (Springer, Berlin, 1980).

${ }^{6}$ N. Woodhouse, Geometric Quantization (Claredon, Oxford, 1980).

${ }^{7}$ M. Puta, Hamiltonian Mechanical Systems and Geometric Quantization, Mathematics and Its Applications, \#260 (Kluwer, Dordrecht, 1993).

${ }^{8}$ A. Echevarría-Enríquez, M. C. Mun̂oz-Lecanda, N. Román-Roy, and C. Victoria-Monge, "Mathematical foundations of geometric quantization (Part 1)," Preprint UPC, 1993.

${ }^{9}$ A. Lichnerowicz, "Les variétés de Poisson et les algébres de Lie associées," J. Diff. Geom. 12, 253-300 (1977).

${ }^{10}$ P. Dazord, A. Lichnerowicz, and Ch. M. Marle, "Structure locale des variétés de Jacobi," J. Math. Pures Appl. 70, 101-152 (1991).

${ }^{11}$ F. Guédira and A. Lichnerowicz, “Géométrie des algébres de Lie locales de Kirillov," J. Math. Pures Appl. 63, 407-484 (1984).

${ }^{12}$ A. Lichnerowicz, "Les variétés de Jacobi et leurs algébres de Lie associées," J. Math. Pures Appl. 57, 453-488 (1978).
} 
${ }^{13}$ E. Getzler, "Batalin-Vilkovisky algebras and two-dimensional topological field theories," Commun. Math. Phys. 159, 265-286 (1994).

${ }^{14}$ A. Ibort, M. de León, and G. Marmo, "Reduction of Jacobi manifolds," J. Phys. A 30, 2783-2798 (1997).

${ }^{15}$ Y. Kosmann-Schwarzbach, "From Poisson algebras to Gerstenhaber algebras,' Centre de Mathématiques, Ecole Polytechnique Preprint, 1995.

${ }^{16}$ E. Witten, “A note on the antibracket formalism,” Mod. Phys. Lett. A 5, 487-494 (1990).

${ }^{17}$ D. Chinea, M. de León, and J. C. Marrero, "Prequantizable Poisson manifolds and Jacobi structures," J. Phys. A 29, 6313-6324 (1996).

${ }^{18}$ Y. Kerbrat and Z. Souici-Benhammadi, "Variétés de Jacobi et groupoides de contact," C. R. Acad. Sci., Ser. I: Math. 317, 81-86 (1993).

${ }^{19}$ M. de León, J. C. Marrero, and E. Padrón, “Lichnerowicz-Jacobi cohomology of Jacobi manifolds,” C. R. Acad. Sci., Ser. I: Math. 324, 71-76 (1997).

${ }^{20}$ M. de León, J. C. Marrero, and E. Padrón, “A generalization for Jacobi manifolds of the Lichnerowicz-Poisson cohomology," in Proceedings of the V Fall Workshop: Differential Geometry and its Applications, Jaca, September 23-25, 1996.

${ }^{21}$ M. de León, J. C. Marrero, and E. Padrón, “Lichnerowicz-Jacobi cohomology," J. Phys. A 30 6029-6055 (1997).

${ }^{22}$ M. de León, J. C. Marrero, and E. Padrón, "H-Chevalley-Eilenberg cohomology of a Jacobi manifold and JacobiChern class,'” C. R. Acad. Sci., Ser. I: Math. 325, 405-410 (1997).

${ }^{23}$ R. W. Urwin, "The prequantization representations of the Poisson Lie algebra," Adv. Math. 50, 126-154 (1983).

${ }^{24}$ M. V. Karasev, "Analogues of objects of Lie group theory for nonlinear Poisson brackets," Math. U.S.S.R. Izv. 28, 497-527 (1987).

${ }^{25}$ A. Weinstein, "Symplectic groupoids and Poisson manifolds,” Bull. Am. Math. Soc. 16, 101-104 (1987).

${ }^{26}$ M. V. Karasev and V. P. Maslov, Nonlinear Poisson Brackets. Geometry and Quantization, Translations of Mathematical Monographs, 119 (A.M.S., Providence, RI, 1993).

${ }^{27}$ I. Vaisman, Lectures on the Geometry of Poisson Manifolds, Progress in Math. 118 (Birkhäuser, Basel, 1994).

${ }^{28}$ P. Dazord, "Intégration d'algèbres de Lie locales et groupoïdes de contact," C. R. Acad. Sci., Ser. I: Math. 320, 959-964 (1995).

${ }^{29}$ K. H. Bhaskara and K. Viswanath, Poisson Algebras and Poisson Manifolds, Research Notes in Mathematics, 174 (Pitman, London, 1988).

${ }^{30}$ A. Kirillov, “Local Lie algebras," Russian Math. Surveys 31, 55-75 (1976).

${ }^{31}$ P. Libermann and Ch. M. Marle, Symplectic Geometry and Analytical Mechanics (Kluwer, Dordrecht, 1987).

${ }^{32}$ A. Weinstein, "The local structure of Poisson manifolds," J. Diff. Geom. 18, 523-557 (1983); 22, 255E (1985).

${ }^{33} \mathrm{C}$. Albert, "Le théorème de réduction de Marsden-Weinstein en géométrie cosymplectique et de contact," J. Geom. Phys. 6(4), 627-649 (1989).

${ }^{34}$ D. E. Blair, Contact Manifolds in Riemannian Geometry, Lecture Notes in Math., 509 (Springer-Verlag, Berlin, 1976).

${ }^{35}$ F. Cantrijn, M. de León, and E. A. Lacomba, "Gradient vector fields on cosymplectic manifolds," J. Phys. A 25 , 175-188 (1992).

${ }^{36}$ I. Vaisman, “Locally conformal symplectic manifolds,” Int. J. Math. Math. Sci. 8(3), 521-536 (1985).

${ }^{37}$ J. Grabowski, G. Marmo, A. Perelomov, and A. Simoni, “'Remarks on Virasoro and Kac-Moody algebras," Int. J. Mod. Phys. A 11, 28, 4969-4984 ( 1996).

${ }^{38}$ Ch. M. Marle, "Quelques propriétés des variétés de Jacobi," in Géométrie Symplectique et Mécanique, Seminaire sud-rhodanien de géométrie, edited by J-P. Dufour (Travaux en Cours, Hermann, Paris, 1985), pp. 125-139.

${ }^{39}$ R. S. Palais, "A global formulation of the Lie theory of transformation groups," Mem. Am. Math. Soc. 22, (1957).

${ }^{40}$ S. Kobayashi, "Principal fibre bundle with 1-dimensional toroidal group," Tôhoku Math. J. 8(2), 29-45 (1956).

${ }^{41}$ J. Pradines, "Théorie de Lie pour les groupoïdes différentiables," C.R. Acad. Sci., Ser. A 263, 907-910 (1966).

${ }^{42}$ K. H. Bhaskara and K. Viswanath, "Calculus on Poisson manifolds," Bull. London Math. Soc. 20, 68-72 (1988)

${ }^{43}$ A. Coste, P. Dazord, and A. Weinstein, “Groupoïdes symplectiques,'” Pub. Dép. Math. Lyon 2/A 1-62 (1987).

${ }^{44}$ I. Vaisman, "Basic ideas of geometric quantization,", Rend. Sem. Mat. Torino 37, 31-41 (1979).

${ }^{45}$ S. L. Woronowicz, “Compact matrix pseudogroups,” Commun. Math. Phys. 111, 613-665 (1987).

${ }^{46}$ J. Grabowski, "Quantum SU(2) group of Woronowicz and Poisson structures," in Differential Geometry and its Applications, Proc. Conf., Aug. 27-Sep. 2, 1989, Brno, Czechoslovaquia (World Scientific, Singapore, 1990), pp. 313-322.

${ }^{47}$ J. Grabowski and P. Urbanski, “Tangent lifts of Poisson and related structures," J. Phys. A 28, 6743-6777 (1995).

${ }^{48}$ I. Vaisman, "On the geometric quantization of the symplectic leaves of Poisson manifolds," Diff. Geom. Appl. 7(3), 265-277 (1997).

${ }^{49}$ S. Sternberg, Lectures on Differential Geometry (Prentice-Hall, Englewood Cliffs, 1964).

${ }^{50}$ M. de León, J. C. Marrero, and E. Padrón, "Complex polarizations on cosymplectic and contact manifolds," work in progress.

${ }^{51}$ R. Ibaǹez, M. de León, J. C. Marrero, and D. Martín de Diego, “Coisotropic and Legendre-Lagrangian submanifolds and conformal Jacobi morphisms,”’ J. Phys. A 30, 5427-5444 (1997). 